\title{
A globally stable algorithm for the integration of high-index differential-algebraic systems
}

\author{
Pierluigi Di Franco, Giordano Scarciotti, Member, IEEE, and Alessandro Astolfi, Fellow, IEEE
}

\begin{abstract}
The problem of constraint stabilization and numerical integration for differential-algebraic systems is addressed using Lyapunov theory. It is observed that the application of stabilization methods which rely on a linear feedback mechanism to nonlinear systems may result in trajectories with finite escape time. To overcome this problem we propose a method based on a nonlinear stabilization mechanism which guarantees the global existence and convergence of the solutions. Discretization schemes, which preserve the properties of the method, are also presented. The results are illustrated by means of the numerical integration of a slider-crank mechanism.
\end{abstract}

Index Terms-Differential-algebraic systems, nonlinear systems, numerical integration, constraint stabilization.

\section{INTRODUCTION}

Differential-algebraic systems (also known as DAE systems, descriptor systems or singular systems) are composite systems of differential and algebraic equations. The mathematical modelling of a multitude of engineering systems, such as mechanical systems [1], chemical processes [2] and electrical networks [3], is straightforward in the differential-algebraic formulation: differential equations can be used to describe each dynamical subsystem independently whereas algebraic equations allow describing phenomena such as environmental and topological constraints, mass and flow conservation, and thermodynamic relations.

Due to the large field of application in which DAE systems are involved, the stability analysis and control of such systems have gained great attention from the research community. In the linear case, DAE systems have been studied in [4], in which several concepts from the theory of dynamical systems have been extended to this class of systems. Then [5] and [6] have provided a Lyapunov-based analysis for linear DAE systems. In [7] the stability analysis of DAE systems has been performed by means of linear matrix inequalities, yielding necessary and sufficient conditions for the linear case and sufficient conditions for the nonlinear case. One of the first studies in the control of nonlinear DAE systems has been presented in [8], in which global stabilization and tracking problems for

P. Di Franco, G. Scarciotti and A. Astolfi are with the Dept. of Electrical and Electronic Engineering, Imperial College London, London, SW7 2AZ, UK, [pierluigi.di-franco13][g.scarciotti][a.astolfi]@ic.ac.uk.

A. Astolfi is also with DICII, University of Rome "Tor Vergata", Via del Politecnico 1, 00133 Rome, Italy.

This work has been partially supported by the European Union's Horizon 2020 Research and Innovation Programme under grant agreement No 739551 (KIOS CoE). constrained mechanical systems have been solved by means of force feedback, while local results have been obtained by means of a linear controller. Many existing control results for DAE systems rely on the derivation of appropriate state-space realizations of the constrained system. For example [9] has studied the feedback stabilization problem for nonlinear DAE systems by means of a change of coordinates which transforms the DAE system in the so-called normal form and local results have been obtained by linearization of the transformed system. Recently, the zero dynamics form for DAE systems has been introduced in [10], see also [11].

Although the modelling process is simplified by the use of differential-algebraic equations, numerous complications arise in their numerical integration ${ }^{1}$. An important concept which provides a measure of these difficulties is the concept of index. Loosely speaking, the index indicates the number of time differentiations required to reduce a DAE system to a system of ordinary differential equations (ODEs), see [12] for a precise definition. While numerical methods for the integration of index-1 DAE systems are well-established, see e.g. [13], [14] and [15], for high-index systems direct integration methods are yet to be developed. Good reviews on some of the approaches to the integration of high-index DAE systems are given in [16] and [17].

Coordinates reduction techniques are used to reduce DAE systems to a minimum set of ODEs, which can then be directly integrated by means of classical methods. Such techniques include Maggi's formulation, see [18], [19] and [20], the Index-1 formulation, see [21], [22], [23] and [24], the nullspace formulation [22], and Udwadia and Kalaba's formulation, see [25] and [26]. However, when numerically integrating the resulting ODEs, the initial conditions must be consistent with the original set of algebraic equations and with a finite number of their time derivatives. In addition, round-off errors introduced by the numerical solver may yield a constraint violation. Since for DAE systems the solution manifold ${ }^{2}$ is invariant but not attractive, see [27], any constraint violation due to inconsistent initial conditions or numerical approximations may make the solution drift away from the manifold: this is called drift phenomenon or constraint drift. Hence, several methods have been proposed to guarantee that the solution remains in (or close to) the solution manifold. The paper [28] has proposed an

\footnotetext{
${ }^{1}$ Depending on the context, we use the term integration to mean either the evaluation of solutions of differential-algebraic equations or the construction of numerical algorithms for such an evaluation.

${ }^{2}$ See Remark 1 for a precise definition.
} 
index reduction technique in combination with the Pantelides's algorithm [29] to find consistent initial values. The result of the reduction process is an augmented, but consistent, index-1 DAE system, which can be directly integrated by means of index-1 methods. Since the required differentiations are carried out analytically rather than numerically, the technique has an accuracy comparable to that of solving a state-space formulation of the problem. However, the reduction comes at the cost of a higher analytical computation due to the higher number of equations and variables involved. Projection methods aim to project the solution onto the solution manifold by means of an iterative process, see [30] and [31]. As a drawback, the adaptive and iterative character of these methods make them unsuited for real-time simulations. To guarantee that the projection step is successfully completed in a priori fixed sampling interval, [32] has proposed and discussed a non-iterative projection strategy yielding a bound for the constraint error which is independent of time. Other approaches, known as stabilization methods, are based on ideas and tools borrowed from control theory. They aim to avoid drift by adding extra terms that, although vanishing on the solution manifold, have the effect of making this manifold asymptotically attractive. The most widely used method of this kind is the Baumgarte's method [33], which is based upon the principle of feeding back a linear combination of all the constraint errors. The choice of the feedback parameters depends on several factors, such as the model and the numerical solver used. A direct comparison of the aforementioned methods, with a list of advantages and disadvantages and a case study, can be found in [34] and [35].

Some attempts to improve Baumgarte's method have been presented in [36], in which an adaptive computation of the feedback parameters has been proposed, or in [37], in which the integral of the constraint violation has been added to the feedback law. A generalization of the Baumgarte's method for nonlinear systems is presented in [38], in which stability properties of the solution manifold along with discretization schemes which preserve such properties have been derived using classical Lyapunov theory. An interpretation of the Baumgarte's method as an output nullifying feedback control has been provided, in the framework of Lie algebraic control theory, in [39]. Recently, [40] has proposed a nonlinear stabilization method for dynamical systems on manifolds which preserves first integrals of the system, such as the kinetic energy or the module of the angular momentum for mechanical systems, for any ordinary numerical integrator. However, stabilization methods which rely on a linear feedback mechanism may lead to "closed-loop systems" with solutions having finite escape time. To the best of our knowledge none of the existing methods addresses this issue.

It is important to observe that stabilization methods add extra terms to the equations which affect the solution's trajectory off the solution manifold. Depending on the stabilization method and on the constraint error, stability properties of the equilibrium points of the DAE system may be changed, resulting in possibly unexpected non-physical behaviour. Moreover, additional instabilities may arise whenever a time discretization of the system is performed, i.e. whenever the integration method is replaced by a numerical algorithm. With Baumgarte's method, for instance, the trajectory of the discretized system may drift away from the solution manifold even though the manifold is asymptotically stable for the underlying ODE system in the continuous-time domain. Thus, it is of interest to ensure that stability properties of the solution manifold and of the equilibrium points are preserved when discretization schemes are applied. It is worth stressing, therefore, that Baumgarte's method, and all its variations, rely on notions (and tools) from control theory, hence improvements can be obtained using ideas and tools borrowed from control theory.

In this paper the problems of constraint stabilization and numerical integration for DAE systems with high-index are addressed by means of concepts borrowed from classical geometric control theory, such as the notions of relative degree and zero dynamics (see [41] for an introduction to these topics), and by means of classical Lyapunov theory. Popular stabilization methods such as Baumgarte's method, or the one in [38], are recast in the proposed framework and are shown to potentially lead to systems exhibiting solutions with finite escape time. On this basis, we propose a nonlinear stabilization method which ensures the existence of the solution for all times. In addition, in the case in which the underlying DAE system has an equilibrium point which is asymptotically stable, we show that the proposed method preserves this property in a neighborhood of the solution manifold. To avoid instabilities due to time discretization we present two discrete-time schemes and conditions to select the sampling time such that the properties of the method are preserved. The results are illustrated via a step-by-step algorithm for the simulation of DAE systems and validated with an example. Preliminary results have been published in [42], [43] and [44]. The additional contributions of the present paper are as follows: the results are presented in a more organized way with formal proofs; two popular stabilization methods have been showed to potentially lead to solutions which may exhibit finite escape time; the simulation algorithm is revisited and complemented by discretization schemes; a new worked-out example with complete calculations and comparisons with two popular stabilization methods is provided.

The rest of the paper is organized as follows. In Section II we recall some general concepts of the theory of DAE systems and we give some preliminary results. In Section III we study the problem of constraint stabilization and main results are derived in the continuous-time domain. In Section IV we present two discretization schemes which preserve the properties highlighted in Section III. Moreover, we summarize the proposed results in an algorithm for the simulation of nonlinear DAE systems. In Section V, the method is validated with an example (a slider-crank mechanism) and simulation results are discussed. Finally Section VI reports our conclusions.

Notation. We use standard notation. The symbols $\mathbb{R}_{\geq 0}$ and $\mathbb{N}_{\geq 0}$ indicate the sets of non-negative real and integer numbers, respectively. The superscript $T$ represents the transposition operator. Given a vector $x$, the symbol $\|x\|$ denotes its $\mathcal{L}^{2}$ norm. Given a map $f: \mathbb{R}^{n} \rightarrow \mathbb{R}$, we use equivalently the symbols $\nabla_{x} f$ and $\frac{\partial f}{\partial x}$ to denote the row vector of partial derivatives of $f$ with respect to the vector $x \in \mathbb{R}^{n}$. The symbol $L_{f} h$ denotes the Lie derivative of the function $h$ along the 
vector field $f$, while $L_{f}^{k} h=L_{f}\left(L_{f}^{k-1} h\right)$, provided all partial derivatives exist. The symbol $y^{(k)}(t)$ indicates the $k$-th time derivative of the function $y$ at time $t$, provided it exists. Given a manifold $\mathcal{Z}$, the symbol $\left.f\right|_{\mathcal{Z}}$ indicates the restriction of $f$ to $\mathcal{Z}$. All functions and mappings are assumed differentiable as many times as needed.

\section{Preliminaries}

The objective of this section is to introduce some preliminaries on DAE systems and define a formalism to study the stability properties of such systems. The framework presented is useful to highlight some limitations of a class of stabilization methods for the integration of DAE systems based on linear control theory and represents the basis to develop nonlinear discretization schemes which remove the aforementioned limitations.

Consider the DAE system in semi-explicit ${ }^{3}$ form

$$
\dot{x}=f(x)+g(x) \lambda, \quad 0=h(x),
$$

where $x(t) \in \mathbb{R}^{n}$ is the state vector, $\lambda(t) \in \mathbb{R}^{m}$ is the algebraic variable, and $f: \mathbb{R}^{n} \rightarrow \mathbb{R}^{n}, g: \mathbb{R}^{n} \rightarrow \mathbb{R}^{n \times m}$ and $h: \mathbb{R}^{n} \rightarrow \mathbb{R}^{m}$ are smooth vector fields and mappings. For simplicity of notation we assume, in the remainder of the paper, that $m=1$, if not stated otherwise. We also assume that the DAE system (1) is solvable in the sense of Definition 2.2.1 in [46]. Note that the form in (1) contains a general class of DAE systems which includes, for example, mechanical systems with holonomic and nonholonomic constraints [47], and chemical systems described by lumped parameters models or via spatial discretization of the underlying partial differential equations [2]. A concept which plays an important role in the classification and behaviour of DAE systems is the one of differentiation index.

Definition 1. [46] The differentiation index $\nu$ of the DAE system (1) at a point $\left(x^{\circ}, \lambda^{\circ}\right)$ is equal to the minimum number of times it is necessary to differentiate all or part of the equations in (1) with respect to $t$ to determine $\dot{x}$ and $\dot{\lambda}$ as continuous functions of $x$ and $\lambda$ in a neighborhood of $\left(x^{\circ}, \lambda^{\circ}\right)$.

Several notions of index have been defined for DAE systems, see e.g. [12] for a detailed explanation. Here we recall the fact that for the DAE system in semi-explicit form (1) the perturbation index, the tractability index and the geometric index are numerically equal to the differentiation index, although these are defined under different technical (smoothness) assumptions. Finally, the strangeness index, when defined, is equal to $\nu-1$. In the remaining of the paper, the term index refers to the differentiation index. Observe that the description (1) represents a class of DAE systems with index $\nu>1$. This is without loss of generality since index-1 DAE systems are structurally similar to ordinary differential systems and numerical methods for their integration are well-established (see [13], [14] and [15]), thus they are not considered in the present study.

To study the problem of stabilization of manifolds it is helpful to consider the DAE system (1) as an input-affine nonlinear system in which the "input" $\lambda(t)$ and the initial

\footnotetext{
${ }^{3}$ See [45] for the transformation of fully-implicit DAE systems to the semi-explicit form in (1) and vice versa.
}

conditions $x\left(t_{0}\right)=x_{0}$ are consistent with the conditions that the "output" $h(x)$ and its time derivative up to the order $\nu-1$, i.e, $y^{(i)}(t)=h^{(i)}(x(t)), i=0, \ldots, \nu-1$, are identically zero for all times. Stabilization of manifolds is a well-established area of research in the framework of differential geometry, see e.g. [41]. In particular, attempts to study DAE systems in this framework have been made in [9], which has studied the feedback stabilization problem for nonlinear DAE systems by means of a change of coordinates which transforms the DAE system in the so-called normal form. With reference to [41], it is convenient to introduce the concept of relative degree, which is here adapted in the context in which the algebraic equation in (1) is considered as an output.

Definition 2. The DAE system (1) is said to have relative degree $r$ at a point $x^{\circ}$ if

(i) $L_{g} L_{f}^{k} h(x)=0$ for all $x$ in a neighborhood of $x^{\circ}$ and all $k<r-1$;

(ii) $L_{g} L_{f}^{r-1} h\left(x^{\circ}\right) \neq 0$.

The following preliminary result shows that there exists a relation between the concepts of index and relative degree.

Lemma 1. Let $\nu$ and $r$ be the index and the relative degree of the DAE system (1), respectively, and assume these are well-defined at $\left(x^{\circ}, \lambda^{\circ}\right)$ and $x^{\circ}$, respectively. Then $\nu=r+1$.

Proof. Assume that system (1) has relative degree $r$ at $x^{\circ}$. Differentiating with respect to time the output $y=h(x)$ yields $y^{(k)}(t)=L_{f}^{k} h(x(t))$ for all $k<r$ and for all $t$ for which $x(t)$ is defined and close to $x^{\circ}$, and

$$
y^{(r)}(0)=L_{f}^{r} h\left(x^{\circ}\right)+L_{g} L_{f}^{r-1} h\left(x^{\circ}\right) \lambda(0) .
$$

Hence, the relative degree $r$ is equal to the number of times the output $y$ has to be differentiated to have the input $\lambda$ explicitly appearing in equation (2). Since $y^{(i)}(t)=0$, for $i \geq 0$ and for all $t$ for which $x(t)$ is defined and close to $x^{\circ}$, then, from (2), we obtain $0=L_{f}^{r} h(x)+L_{g} L_{f}^{r-1} h(x) \lambda$ and, since $L_{g} L_{f}^{r-1} h(x) \neq 0$ in a neighborhood of $x^{\circ}$, we can compute $\lambda$ as a function of $x$, i.e.

$$
\lambda=-\left(L_{g} L_{f}^{r-1} h(x)\right)^{-1} L_{f}^{r} h(x)
$$

and $\lambda^{\circ}=-\left(L_{g} L_{f}^{r-1} h\left(x^{\circ}\right)\right)^{-1} L_{f}^{r} h\left(x^{\circ}\right)$. Differentiating once more equation (3) we obtain the expression of $\dot{\lambda}$ as function of $x$ and $\lambda$, proving the claim.

The existence of a well-defined relative degree for the DAE system (1) implies the existence of a local diffeomorphism which induces on system (1) a special structure, the so-called normal form [41], as described in the following statement. Note also that we remove, for simplicity, reference to the points $\left(x^{\circ}, \lambda^{\circ}\right)$ or $x^{\circ}$. All statements are however locally defined around a point $\left(x^{\circ}, \lambda^{\circ}\right)$ or $x^{\circ}$.

Proposition 1. Consider the system (1) and assume it has index $\nu=r+1, r \in \mathbb{N}_{>0}$. Consider the mapping $z=\Phi(x)$, defined as

$$
\Phi(x)=\left(h(x), L_{f} h(x), \ldots, L_{f}^{r-1} h(x), \phi_{r+1}, \ldots, \phi_{n}\right)^{\top},
$$


in which the mappings $\phi_{i}: \mathbb{R}^{n} \rightarrow \mathbb{R}$, for $i=r+1, \ldots, n$ are such that the Jacobian of $\Phi$ is invertible and $L_{g} \phi_{i}=0$. Then system (1) can be written in the coordinates $z=\left(\xi^{\top}, \eta^{\top}\right)^{\top}=$ $\Phi(x)$, as

$$
\begin{aligned}
\xi_{1} & =0, \dot{\xi}_{1}=\xi_{2}, \ldots, \dot{\xi}_{r-1}=\xi_{r}, \dot{\xi}_{r}=b(\xi, \eta)+a(\xi, \eta) \lambda \\
\dot{\eta} & =q(\xi, \eta)
\end{aligned}
$$

where $\xi_{i}(t) \in \mathbb{R}$, for $i=1, \ldots, r, \xi=\left(\xi_{1}, \ldots, \xi_{r}\right)^{\top}, \eta(t) \in$ $\mathbb{R}^{n-r}$ and $a, b$ and $q$ are smooth mappings such that $a(0,0) \neq 0$.

Proof. Observe that, by definition, the algebraic subsystem of (1) can be written in the coordinates $z=\Phi(x)$ as $z_{1}=$ $\xi_{1}=0$. On the other hand, the differential subsystem of (1) can be written as the one in (5), see Proposition 4.1.3 in [41]. Therefore, the claim follows by appending the algebraic equation to the differential subsystem.

Remark 1. The solution manifold of system (5) is

$$
\mathcal{M}=\left\{\left(\xi^{\top}, \eta^{\top}\right)^{\top}: \xi=0\right\},
$$

and there exists a unique smooth mapping

$$
\lambda(\xi, \eta)=-\frac{b(\xi, \eta)}{a(\xi, \eta)},
$$

such that $\dot{\xi}_{r}(t)=0$ for all $t$, which coincides with equation (3) expressed in the coordinates $z=\left(\xi^{\top}, \eta^{\top}\right)^{\top}$ defined in Proposition 1. Note also that the $\eta$ subsystem of (5) restricted to the solution manifold, i.e.

$$
\dot{\eta}=q(0, \eta)
$$

coincides with the zero dynamics of the auxiliary system $\dot{x}=$ $f(x)+g(x) \lambda$ with input $\lambda$ and output $y=h(x)$. We therefore refer to (8) as the zero dynamics of the DAE system (1).

A direct consequence of the reformulation in Proposition 1 is that the stability properties of the equilibrium points of (1) can be studied in the special form (5), as shown in the following theorem.

Theorem 1. Suppose that the DAE system (1) has index $1<\nu \leq n$. Stability properties, see [48, Definition 2.3], of the equilibrium points of system (1) are equivalent to stability properties of the zero equilibrium of the zero dynamics (8).

Proof. From Proposition 1 there exists a change of coordinates which transforms system (1) in the normal form (5). In particular, it is always possible to choose the coordinates $\Phi(x)=$ $\left(\xi_{1}, \ldots, \xi_{r}, \eta\right)$ such that $\Phi\left(x^{\circ}\right)=\left(\xi_{1}^{\circ} \ldots \xi_{r}^{\circ}\left(\eta^{\circ}\right)^{\top}\right)^{\top}=0$.

Note now that for any $\left(\xi(0)^{\top}, \eta(0)^{\top}\right)^{\top} \in \mathcal{M}$, where $\mathcal{M}$ is given in (6), $\xi_{i}(t)=0$ for all $t \geq 0$, for $i=1, \ldots, r$, while the subsystem of (5) in the variable $\eta$ reduces to the zero dynamics (8). As a consequence, the stability properties of the equilibrium point $x^{\circ}$ are equivalent to the stability properties of the equilibrium point $\eta^{\circ}$, proving the claim.

Remark 2. System (8) has dimension equal to (from Lemma 1) $n-r=n-\nu+1$, where $\nu-1$ is the actual ${ }^{4}$ number of constraints acting on the system. Thus (8) is a local representation of system (1) with the minimum number of independent coordinates.

Remark 3. The case $\nu>n$ implies the existence of at least $n$ constraints acting on the system. This means that the state belongs (generically) to a zero dimensional manifold, or the equations have no solution.

Remark 4. In the general case in which $m>1$, the existence of a well-defined (vector) relative degree cannot be inferred from the well-definiteness of the differential index. However, if $x^{\circ}$ is a regular point of the zero dynamics algorithm [41], the application of such algorithm leads to the calculation of a maximal zeroing submanifold onto which a zero dynamics can be defined. Moreover, the steps of the zero dynamics algorithm are helpful in defining a local diffeomorphism which transforms the DAE system (1) in the so-called global normal form, which reduces to the form (5) in the case $m=1$. Although, in general, the algebraic variable appears explicitly in the $\eta$ subsystem of (5), there always exists a $\operatorname{smooth}^{5} \lambda=\lambda^{*}(\xi, \eta)$, solution of the system of equations $\dot{\xi}=0$, which renders the $\eta$ subsystem function of $\xi$ and $\eta$ only, namely $\dot{\eta}=q(\xi, \eta)$. If, in addition, the (vector) relative degree is well defined, then the mapping $\lambda^{*}(\xi, \eta)$ is unique.

\section{CONSTRAINT STABILIZATION}

The objective of this section is to address the problem of integration of DAE systems. Typical problems arising when DAE systems are numerically integrated include inconsistent initial conditions, round-off errors and constraint drift. Popular methods to avoid these issues, such as Baumgarte's method [33], rely on ideas borrowed from linear feedback theory. We show that the application of such methods may lead to systems with solutions having finite escape time. Nonlinear "stabilizer" are then designed to cope with this problem. Moreover, in the case in which an equilibrium point of the DAE system is asymptotically stable, the proposed method preserves this property in a neighborhood of the solution manifold. All the aforementioned problems and objectives are recast within the formalism presented in Section II and are studied, initially, in the continuous-time domain.

Consider the DAE system (5) with index $\nu=r+1, r \in \mathbb{R}_{>0}$. Usual index reduction techniques (see [21], [22] or [25]) would normally impose $\dot{\xi}_{r}=0$ and directly integrate the differential subsystem in (5). However, the solution of (5), when $\dot{\xi}_{r}=0$ and the equation $\xi_{1}=0$ is disregarded, is such that $\xi_{1}(t)=$ $\sum_{i=1}^{r} \frac{t^{i-1}}{(i-1) !} \xi_{i}(\bar{t})$ for all $t \geq \bar{t}$ : it is clear that the violation of the constraints would grow unbounded if $r>1$. This describes the drift phenomenon or constraint drift and reflects the property of the manifold $\mathcal{M}$ of being invariant but not attractive, see [27].

\footnotetext{
${ }^{4}$ This includes the "hidden" constraints which can be revealed by repeated time-differentiations of the original set of algebraic equations.

${ }^{5}$ In the general case in which $m>1$, we use the symbol $\xi$ to indicate the column vector $\operatorname{col}\left(\xi^{1}, \ldots, \xi^{m}\right)$, in which $\xi^{j}=\operatorname{col}\left(\xi_{1}^{j}, \ldots, \xi_{r_{j}}^{j}\right), \xi_{i}^{j} \in \mathbb{R}$ for $i=1, \ldots, r_{j}$ and $j=1, \ldots, m, r_{j}=\nu_{j}-1$ and $\nu_{j}$ is the index of the $j$-th component of the vector $h(x)$. However, with the aim of maintaining the notation simple, we drop the superscript $j$ for the case $m=1$.
} 
Stabilization methods aim to cope with the constraint drift by feeding back the constraint violation. In general, stabilization methods based on linear feedback directly integrate differential systems described by equations of the form

$$
\dot{\xi}=A \xi, \quad \dot{\eta}=q(\xi, \eta),
$$

in place of system (5), where $A \in \mathbb{R}^{r \times r}$ is Hurwitz. A popular stabilization technique which is consistent with the above structure is Baumgarte's [33], which replaces the equation $\dot{\xi}_{r}=0$ with the equation

$$
\dot{\xi}_{r}=-\sum_{j=1}^{r-1} \alpha_{j} \xi_{j}
$$

in which the coefficients $\alpha_{j}$, for $j=1, \ldots, r$, are such that the roots of the polynomial $\sigma(\tau)=\tau^{r}+\sum_{j=1}^{r-1} \alpha_{j} \tau^{j}$, in the indeterminate $\tau$ have all negative real part. Despite the conceptual simplicity of such a method, this may be inadequate for nonlinear DAE systems. To see this point, consider the following example.

Example 1. Consider the DAE system with a linear stabilizer a-la Baumgarte described by the equations

$$
\xi_{1}=0, \quad \dot{\xi}_{1}=-\beta \xi_{1}, \quad \dot{\eta}=-\eta+\xi_{1} \eta^{2},
$$

with $\beta>0$. Note that if $\xi_{1}(0) \neq 0$ then the $\eta$ subsystem becomes $\dot{\eta}=-\eta+\xi_{1}(0) e^{-\beta t} \eta^{2}$, from which it is clear that for any $\beta>0$ there exists a scalar $M>0$ such that, for all $\eta(0)>M$, the state $\eta(t)$ escapes to infinity in finite time. This implies that the use of linear stabilization methods for nonlinear DAE systems may be inadequate.

Remark 5. The finite escape time of solutions may arise even with stabilization methods specifically designed for nonlinear DAE systems. For instance, a generalization of Baumgarte's method for nonlinear systems is presented in [38] $]^{6}$. This method can be described, in some cases, by equations of the form (9). To see this, consider the reduced index-1 DAE system

$$
\dot{x}=\widehat{f}(x), 0=\widehat{h}(x),
$$

where the mapping $\widehat{f}$ has been obtained from the differential subsystem in (1) with the algebraic variable eliminated, i.e.

$$
\widehat{f}(x)=f(x)+g(x) \lambda^{*}(x),
$$

with $\lambda^{*}(x)$ given in (3) and

$$
\widehat{h}(x)=\left[\begin{array}{lll}
h(x) & \ldots & L_{\widehat{f}}^{r-1} h(x)
\end{array}\right]^{\top} .
$$

The reference [38] considers the family of stabilization methods given by

$$
\dot{x}=\widehat{f}(x)-\gamma F(x) \widehat{h}(x),
$$

where $\gamma>0$,

$$
F(x)=D(x)(C(x) D(x))^{-1}, \quad C(x)=\frac{\partial \widehat{h}(x)}{\partial x},
$$

and $D(x)$ chosen such that $C(x) D(x)$ is nonsingular, e.g.

\footnotetext{
${ }^{6}$ Note that this is employed in the popular software MathWorks Multibody ${ }^{\mathrm{TM}}$ (formerly SimMechanics ${ }^{\mathrm{TM}}$ ) for real-time simulations, see [49].
}

$D(x)=C^{\top}(x)$ (observe that $C(x)$ is full-rank). Note that system (15) can be expressed in the coordinates $z=(\xi, \eta)=\Phi(x)$ as

$$
\begin{aligned}
\dot{z}= & {\left.\left[\nabla_{x} \Phi(x) \dot{x}\right]\right|_{x=\Phi^{-1}(z)}=\left[\nabla_{x} \Phi(x)(f(x)+\right.} \\
& \left.\left.+g(x) \lambda^{*}(x)\right)-\gamma \nabla_{x} \Phi(x) F(x) \widehat{h}(x)\right]\left.\right|_{x=\Phi^{-1}(z)} .
\end{aligned}
$$

Let $\nabla_{x} \Phi(x)=\left[\begin{array}{ll}\bar{J}^{\top}(x) & \widehat{J}^{\top}(x)\end{array}\right]^{\top}$, where $\bar{J}: \mathbb{R}^{n} \rightarrow \mathbb{R}^{r \times n}$ is the submatrix composed of the first $r$ rows of $\nabla_{x} \Phi(x)$ while $\widehat{J}: \mathbb{R}^{n} \rightarrow \mathbb{R}^{n-r \times n}$ is the submatrix composed of the last $n-r$ rows of $\nabla_{x} \Phi(x)$. Since $\widehat{h}\left(\Phi^{-1}(\xi, \eta)\right)=\xi$, and $\lambda^{*}\left(\Phi^{-1}(\xi, \eta)\right)$ is such that (7) holds, then system (17) in the coordinates $z=(\xi, \eta)$ becomes

$$
\begin{aligned}
& \dot{\xi}=R \xi-\gamma \bar{J}\left(\Phi^{-1}(\xi, \eta)\right) F\left(\Phi^{-1}(\xi, \eta)\right) \xi \\
& \dot{\eta}=\widetilde{q}(\xi, \eta)-\gamma \widehat{J}\left(\Phi^{-1}(\xi, \eta)\right) F\left(\Phi^{-1}(\xi, \eta)\right) \xi
\end{aligned}
$$

where $\widetilde{q}: \mathbb{R}^{n} \rightarrow \mathbb{R}^{n-r}$ and

$$
R=\left(\begin{array}{ccccc}
0 & 1 & 0 & \ldots & 0 \\
0 & 0 & 1 & \ddots & \vdots \\
\vdots & \vdots & \ddots & \ddots & 0 \\
& & & & 1 \\
0 & 0 & \ldots & & 0
\end{array}\right)
$$

Note now that in the special case in which the mapping $\widehat{h}(x)$ is linear, which is typical for instance in mechanical systems with linear holonomic constraints (see the example in Section VI), it follows from equations (16) that the matrix $F(x)$ is constant. Moreover, the matrix $\bar{J}(\xi, \eta)$ is constant by definition thus, as a consequence, system (18) can be described by equations of the form (9), with $A=R-\gamma \bar{J} F$ and $q(\xi, \eta)=\widetilde{q}(\xi, \eta)+$ $-\gamma \widehat{J}\left(\Phi^{-1}(\xi, \eta)\right) F \xi$, proving that the solutions arising from the method proposed in [38] may result in trajectories which may have finite escape time.

Remark 6. If $\xi(t) \neq 0$ for some $t$ the trajectory of system (9) is different from the trajectory of the DAE system (5). Indeed, under the stated smoothness assumptions, the $\eta$ subsystem of (9) can be rewritten as ${ }^{7}$

$$
\dot{\eta}=q(\xi, \eta)=q_{0}(\eta)+\sum_{i=1}^{r} q_{i}(\xi, \eta) \xi_{i},
$$

where $q_{0}(\eta)=q(0, \eta)$ is the mapping in (8) and $q_{i}: \mathbb{R}^{r} \times$ $\mathbb{R}^{n-r} \rightarrow \mathbb{R}^{n-r}$, for $i=1, \ldots, r$ are smooth mappings. From equation (20) it is clear that if $\xi(\bar{t}) \neq 0$ for some $\bar{t} \geq 0$ the solution $\eta(t)$, for $t \geq \bar{t}$, is affected by an external disturbance which depends on the mappings $q_{i}$ for $i=1, \ldots, r$ and on the matrix $A$ in (9). Observe that not only such disturbances may lead to the finite escape time phenomenon in the worst case scenario, but they may also "destabilize" trajectories which are supposed to converge to an equilibrium. Based on this observation, for the special case in which the DAE system (1) has an asymptotically stable equilibrium point, an additional objective of the stabilization method is to preserve the convergence of the trajectories initialized in a neighborhood of the solution manifold.

\footnotetext{
${ }^{7}$ See Hadamard's Lemma [50].
} 


\section{A. Nonlinear stabilization}

Motivated by the previous considerations we present a nonlinear version of the method captured by system (9) overcoming the indicated limitations. Consider the system

$$
\dot{\xi}_{i}=k_{i}(\xi, \eta) \xi_{i}, \quad \dot{\eta}=q_{0}(\eta)+\sum_{i=1}^{r} q_{i}(\xi, \eta) \xi_{i}
$$

where $\xi_{i}(t) \in \mathbb{R}$, for $i=1, \ldots, r, \eta(t) \in \mathbb{R}^{n-r}, \xi=$ $\left(\xi_{1}, \ldots, \xi_{r}\right)^{\top}, q_{0}$ and $q_{i}$, for $i=1, \ldots, r$, defined in (20) and

$$
k_{i}(\xi, \eta)=-\frac{\delta^{2}}{2}\left\|q_{i}(\xi, \eta)\right\|^{2}-\epsilon,
$$

for $i=1, \ldots, r$, with $\epsilon>0$ and $\delta>0$. For such a system the following result holds.

Lemma 2. Consider systems (5) and (21). Assume system (5) has index $\nu=r+1$, with $r \in \mathbb{N}_{>0}$. Suppose $(\xi(0), \eta(0))$ belongs to the solution manifold $\mathcal{M}$ defined in (6). Then any solution of (5) is a solution of (21) and vice versa.

Proof. On the solution manifold $\mathcal{M}$ of system (5) the equalities

$$
\xi=0, \lambda=-\frac{b(0, \eta)}{a(0, \eta)},
$$

hold, see equation (7). Note now that

$$
q(\xi, \eta)=q_{0}(\eta)+\sum_{i=1}^{r} q_{i}(\xi, \eta) \xi_{i}
$$

with $q_{0}(\eta)=q(0, \eta)$. Then, by replacing (23) and (24) in (5) and (23) in (21) yields the claim.

Since the modified system (21) produces the same trajectory as (5) on the manifold $\mathcal{M}$, we can legitimately modify the dynamics outside $\mathcal{M}$ to render $\mathcal{M}$ attractive. To this end we state the main result of this section.

Theorem 2. Consider system (21). Assume there exists a positive definite and radially unbounded function $W: \mathbb{R}^{n-r} \rightarrow \mathbb{R}$ such that

$$
\frac{\partial W(\eta)}{\partial \eta} q_{0}(\eta) \leq \gamma W(\eta)+\gamma_{0}
$$

for some $\gamma \in \mathbb{R}$ and $\gamma_{0} \in \mathbb{R}$, and

$$
\sup _{\eta \in \mathbb{R}^{n-r}} \frac{\left\|\frac{\partial W(\eta)}{\partial \eta}\right\|^{2}}{W(\eta)} \leq \bar{W}<+\infty
$$

for some $\bar{W}>0$. Then there exist $\bar{\delta}>0$ and $\bar{\epsilon}>0$ such that for all $\delta \geq \bar{\delta}$ and $\epsilon \geq \bar{\epsilon}$ the following statements hold.

1) $\eta(t)$ and $\xi(t)$ exist for all $\eta(0) \in \mathbb{R}^{n-r}, \xi(0) \in \mathbb{R}^{r}$ and $t \geq 0$;

2) $\lim _{t \rightarrow \infty} \xi(t)=0$, for all $\xi(0) \in \mathbb{R}^{r}$.

Proof. To prove claim 1) consider the positive definite function

$$
V(\xi, \eta)=W(\eta)+\sum_{i=1}^{r} \frac{\xi_{i}^{2}}{2} .
$$

Its time derivative along the trajectories of the system is

$$
\dot{V}(\xi, \eta) \leq \frac{\partial W(\eta)}{\partial \eta} q_{0}(\eta)+\frac{1}{2 \delta^{2}}\left\|\frac{\partial W(\eta)}{\partial \eta}\right\|^{2}+
$$

$$
+\frac{\delta^{2}}{2} \sum_{i=1}^{r}\left\|q_{i}(\xi, \eta)\right\|^{2} \xi_{i}^{2}+\sum_{i=1}^{r} k_{i}(\xi, \eta) \xi_{i}^{2},
$$

for any $\delta>0$ and, by replacing the expression of $k_{i}(\xi, \eta)$ given in (22),

$$
\dot{V}(\xi, \eta) \leq \frac{\partial W(\eta)}{\partial \eta} q_{0}(\eta)+\frac{1}{2 \delta^{2}}\left\|\frac{\partial W(\eta)}{\partial \eta}\right\|^{2}-\epsilon \sum_{i=1}^{r} \xi_{i}^{2} .
$$

Note now that under assumption (26) it is always possible to choose $\bar{\delta}$ such that

$$
\bar{\delta}^{2}=\frac{1}{2 \beta} \bar{W},
$$

where $\beta>0$. Without loss of generality, assume that $\gamma>0$ in equation (25). Hence, using assumption (25) in equation (28), for any $\delta \geq \bar{\delta}$ and $\epsilon \geq 0$, yields

$$
\begin{aligned}
& \dot{V}(\xi, \eta) \leq \frac{\partial W(\eta)}{\partial \eta} q_{0}(\eta)+\beta W(\eta)-\epsilon \sum_{i=1}^{r} \xi_{i}^{2} \leq W(\eta)(\beta+\gamma)+\gamma_{0}+ \\
& -\epsilon \sum_{i=1}^{r} \xi_{i}^{2}<\left(W(\eta)+\sum_{i=0}^{r} \frac{\xi_{i}^{2}}{2}\right)(\beta+\gamma)+\gamma_{0}=(\beta+\gamma) V(\xi, \eta)+\gamma_{0} .
\end{aligned}
$$

From the last inequality it follows that $V(\xi, \eta) \leq$ $V(\xi(0), \eta(0)) e^{(\beta+\gamma) t}+\gamma_{0} t$, from which it is clear that $V(\xi, \eta)$ exists for all $t \geq 0$. As a consequence also $\xi(t)$ and $\eta(t)$ exist for all $t \geq 0$, proving claim 1 ). To prove claim 2 ) consider the Lyapunov function candidate

$$
\widetilde{V}(\xi)=\sum_{i=1}^{r} \frac{\xi_{i}^{2}}{2}
$$

and the $\xi$ subsystem of (21). The time derivative of the Lyapunov function along the trajectories of the $\xi$ subsystem is $\dot{\widetilde{V}}(\xi, \eta)=\sum_{i=1}^{r} k_{i}(\xi, \eta) \xi_{i}^{2}=-\frac{\delta^{2}}{2} \sum_{i=1}^{r}\left\|q_{i}(\xi, \eta)\right\|^{2} \xi_{i}^{2}-\epsilon \xi_{i}^{2}<0$,

for all $\xi_{i} \neq 0$, thus proving claim 2). Note that the $\xi$ subsystem is well-defined by claim 1 ).

The global results of Theorem 2 are indeed conceptually appealing but require the explicit knowledge of a function $W$ which satisfies assumptions (25) and (26) globally. This difficulty can be partially overcome if instead of achieving global results one is interested in local results. For the case in which $\gamma<0$ and $\gamma_{0}=0$ in equation (25), the following result shows that the statements of Theorem 2 may be recast in a local fashion. Moreover, an additional insight on the stability properties of the $\eta$ subsystem is given.

Theorem 3. Consider system (21). Let $\mathcal{B} \subseteq \mathbb{R}^{n-r}$ be a closed set which contains the origin. Assume there exists a positive definite function $W$ such that equation (25) holds for all $\eta \in \mathcal{B}$ and for some $\gamma<0$ and $\gamma_{0}=0$, and

$$
\max _{\eta \in \mathcal{B}} \frac{\left\|\frac{\partial W(\eta)}{\partial \eta}\right\|^{2}}{W(\eta)} \leq \bar{W}<+\infty
$$

for some $\bar{W}>0$. Then there exists a neighborhood of the origin $U \subseteq \mathbb{R}^{n}$ and $\bar{\delta}>0, \bar{\epsilon}>0$ such that for all $\delta \geq \bar{\delta}$ and 
$\epsilon \geq \bar{\epsilon}$ the following statements hold.

1) $\eta(t)$ and $\xi(t)$ exist for all $(\eta(0), \xi(0)) \in U$ and $t \geq 0$;

2) $\lim _{t \rightarrow \infty} \xi(t)=0$, for all $(\eta(0), \xi(0)) \in U$;

3) $\lim _{t \rightarrow \infty} \eta(t)=0$, for all $(\eta(0), \xi(0)) \in U$.

Proof. The proof of claim 1) follows the same steps of the proof of Theorem 2. Let $\beta<-\gamma$ in equation (29) and $\bar{\epsilon} \geq-\gamma-\beta$, then equation (30) yields, locally,

$$
\dot{V}(\xi, \eta)<(\beta+\gamma) V(\xi, \eta),
$$

and $V(\xi, \eta)<V(\xi(0), \eta(0)) e^{(\beta+\gamma) t}$, from which it is clear that, since $(\eta(t), \xi(t)) \in U$ for all $t \geq 0$, then $V(\xi, \eta)$ exists for all $t \geq 0$. As a consequence also $\xi(t)$ and $\eta(t)$ exist for all $t \geq 0$, proving claim 1). Since $\beta+\gamma<0$, the proof of claims 2) and 3) directly follow from equation (33).

Remark 7. If $\mathcal{B}=\mathbb{R}^{n-r}$ and $W(\eta)$ is radially unbounded then statements 1), 2) and 3) in Theorem 3 hold globally.

Remark 8. For the general case in which $m>1$ and provided that the DAE system (1) has a well-defined (vector) relative degree, we consider the system

$$
\begin{aligned}
\dot{\xi}_{i}^{j} & =k_{i}^{j}(\xi, \eta) \xi_{i}^{j}, \quad j=1, \ldots, m, \quad i=1, \ldots, r_{j}, \\
\dot{\eta} & =q_{0}(\eta)+\sum_{j=1}^{m} \sum_{i=1}^{r_{j}} q_{i}^{j}(\xi, \eta) \xi_{i}^{j}
\end{aligned}
$$

in place of (21), where $q_{i}^{j}$, for $i=1, \ldots, r_{j}$ and $j=1, \ldots, m$, are smooth mappings and

$$
k_{i}^{j}(\xi, \eta)=-\frac{\delta^{2}}{2}\left\|q_{i}^{j}(\xi, \eta)\right\|^{2}-\epsilon .
$$

Observe also that the claims of Theorems 2 and 3 remain valid. The proof follows the same steps as the case $m=1$, in which the functions (27) and (31) are replaced by

$V(\xi, \eta)=W(\eta)+\sum_{j=1}^{m} \sum_{i=1}^{r_{j}} \frac{\left(\xi_{i}^{j}\right)^{2}}{2}$ and $\widetilde{V}(\xi)=\sum_{j=1}^{m} \sum_{i=1}^{r_{j}} \frac{\left(\xi_{i}^{j}\right)^{2}}{2}$.

Note that if stability properties of the DAE system (1) are known in advance, these can be exploited to verify condition (25), as stated in the following corollary.

Corollary 1. If the equilibrium point $x^{\circ}$ is a globally asymptotically stable equilibrium point for system (1) then condition (25) of Theorem 2 is satisfied with strict inequality for $\gamma_{0}=0$ and any $\gamma \geq 0$.

Proof. If the equilibrium point $x^{\circ}$ of system (1) is globally asymptotically stable then the equilibrium point $\eta^{\circ}$ of the subsystem $\dot{\eta}=q(0, \eta)$ is globally asymptotically stable, see Theorem 1. Hence, there exists a Lyapunov function $W(\eta)$ such that $\dot{W}(\eta)=\frac{\partial W(\eta)}{\partial \eta} q(0, \eta)<0$ for all $\eta \in \mathbb{R}^{n-r}$, proving the claim.

Remark 9. The algebraic variable $\lambda$ can be recovered by observing that the $\xi_{r}$ equation in $(21)$ is equal to the $\xi_{r}$ equation in $(5)$ with $\lambda(\xi, \eta)=\frac{k_{r}(\xi, \eta) \xi_{r}-b(\xi, \eta)}{a(\xi, \eta)}$. Observe also that the previous expression coincides with (7) on the solution manifold $\mathcal{M}$.
The assumptions of Theorems 2 and 3 and the stabilizer (22) require the explicit knowledge of the mappings $q_{0}(\eta)$ and $q_{i}(\xi, \eta)$, for $i=1, \ldots, r$, which in turn require the calculation of the inverse mapping $x=\Phi^{-1}(z)$. These steps can be avoided as indicated in the following statements.

Lemma 3. The claims of Theorems 2 and 3 remain valid if the condition (25) is replaced by

$$
\frac{\partial W(\eta)}{\partial \eta}\left(q(\xi, \eta)-\sum_{i=1}^{r} p_{i}(\xi, \eta) \xi_{i}\right) \leq \gamma W(\eta)+\gamma_{0},
$$

for some $p_{i}: \mathbb{R}^{r} \times \mathbb{R}^{n-r} \rightarrow \mathbb{R}^{n-r}$, and the gains (22) are replaced by

$$
k_{i}(\xi, \eta)=-\frac{\delta^{2}}{2}\left\|p_{i}(\xi, \eta)\right\|^{2}-\epsilon .
$$

Proof. Consider the positive definite function (27). Its time derivative along the trajectory of system (21) can be written as

$\dot{V}(\xi, \eta)=\frac{\partial W(\eta)}{\partial \eta} \widehat{q}(\xi, \eta)+\frac{\partial W(\eta)}{\partial \eta} \sum_{i=1}^{r} p_{i}(\xi, \eta) \xi_{i}+\sum_{i=1}^{r} k_{i}(\xi, \eta) \xi_{i}^{2}$,

where $\widehat{q}(\xi, \eta)=q(\xi, \eta)-\sum_{i=1}^{r} p_{i}(\xi, \eta) \xi_{i}$. The rest of the proof follows the same steps of the proof of Theorems 2 and 3 , in which $q_{0}(\eta)$ and $q_{i}(\xi, \eta)$, for $i=1, \ldots, r$, are replaced by $\widehat{q}(\xi, \eta)$ and $p_{i}(\xi, \eta)$, respectively.

Remark 10. The condition (35) does not require the calculation of the zero dynamics $q_{0}(\eta)$. However, observe that if the mappings $p_{i}(\xi, \eta)$ are equal to $q_{i}(\xi, \eta)$ then equations $(35)$ and (36) reduce to (25) and (22), respectively.

The formulation in (21) still requires the inversion $x=$ $\Phi^{-1}(\xi, \eta)$. In the next result, we show that the results in Theorems 2 and 3 can be derived without inverting any coordinate transformation. To this end, let

$$
\Phi(x)=\left(\chi(x)^{\top} \psi(x)^{\top}\right)^{\top},
$$

where $\chi(x)=\left(h(x), L_{f} h(x), \ldots, L_{f}^{r-1} h(x)\right)^{\top}$ and $\psi(x)=\left(\phi_{r+1}, \ldots, \phi_{n}\right)^{\top}$, in which the mappings $\phi_{i}: \mathbb{R}^{n} \rightarrow$ $\mathbb{R}$, for $i=r+1, \ldots, n$ are such that the Jacobian of $\Phi$ is invertible and $L_{g} \phi_{i}=0$. Consider the nonlinear system

$$
\dot{x}=\widehat{f}(x)+c(x),
$$

with $\widehat{f}(x)$ given in $(13)$ and

$$
c(x)=\left(\frac{\partial \Phi(x)}{\partial x}\right)^{-1}\left(\begin{array}{c}
(S(x)-R) \chi(x) \\
0
\end{array}\right),
$$

in which

$$
S(x)=\operatorname{diag}\left(\begin{array}{c}
-\frac{\delta^{2}}{2}\left\|\nabla_{x} \psi(x) s_{1}(x)\right\|^{2}-\epsilon \\
\cdots \\
-\frac{\delta^{2}}{2}\left\|\nabla_{x} \psi(x) s_{r}(x)\right\|^{2}-\epsilon
\end{array}\right),
$$

$\delta>0, \epsilon>0$ and $s_{i}: \mathbb{R}^{n} \rightarrow \mathbb{R}^{n}$, for $i=1, \ldots, r$ to be defined and $R$ given in (19). Then the following result holds.

Theorem 4. Consider the nonlinear system (38). Assume there exist mappings $\psi$ and $s_{i}$, for $i=1, \ldots, r$, and a positive 
definite and radially unbounded function $W(\psi(x))$ such that the Jacobian of $\Phi$ given in (37) is invertible and

$$
\begin{aligned}
& \frac{\partial W(\psi(x))}{\partial x}\left(\widehat{f}(x)-\sum_{i=1}^{r} s_{i}(x) L_{f}^{i-1} h(x)\right) \leq \\
& \leq \gamma W(\psi(x))+\gamma_{0},
\end{aligned}
$$

for some $\gamma \in \mathbb{R}$ and $\gamma_{0} \in \mathbb{R}$, and

$$
\sup _{x \in \mathbb{R}^{n}} \frac{\left\|\frac{\partial W(\psi(x))}{\partial \psi(x)}\right\|^{2}}{W(\psi(x))} \leq \bar{W}<+\infty,
$$

for some $\bar{W}>0$. Then there exist $\bar{\delta}>0$ and $\bar{\epsilon}>0$ such that, for all $\delta \geq \bar{\delta}$ and $\epsilon \geq \bar{\epsilon}$, the following statements hold.

1) $x(t)$ exists for all $x(0) \in \mathbb{R}^{n}$ and for all $t \geq 0$;

2) $\lim _{t \rightarrow \infty} h^{(i)}(x(t))=0$, for $i=0,1, \ldots, r-1$.

In addition, if $\gamma<0$ and $\gamma_{0}=0$ then there exist $\bar{\delta}>0$ and $\bar{\epsilon}>0$ such that for all $\delta \geq \bar{\delta}$ and $\epsilon \geq \bar{\epsilon}$ in (40) the following statement holds.

3) $\lim _{t \rightarrow \infty} x(t)=x^{\circ}$, for all $x(0) \in \mathbb{R}^{n}$.

Proof. The proof of the claims is based on the fact that system (38) is described in the coordinates $z=\Phi(x)$ by equations (21)-(36), for which all the statements hold. Differentiating $z=\Phi(x)$ with respect to time we obtain $\dot{z}=\nabla_{x} \Phi(x) \dot{x}$ and, by replacing (38) in the previous equation, yields

$$
\dot{z}=\nabla_{x} \Phi(x) \widehat{f}(x)+\nabla_{x} \Phi(x) c(x) .
$$

Note now that by applying the coordinate transformation $x=\Phi^{-1}(z)$ to the first term of (43) yields the differential subsystem in (5), in which $\dot{\xi}_{r}=$ 0 , i.e. $\left.\left(\nabla_{x} \Phi(x) f(x)+\nabla_{x} \Phi(x) g(x) \lambda^{*}(x)\right)\right|_{x=\Phi^{-1}(z)}=$ $\left(\begin{array}{c}R \xi \\ q(\xi, \eta)\end{array}\right)=B(\xi, \eta)$. Replacing (39) in the second term of (43) and by applying the inverse coordinate transformation $x=\Phi^{-1}(z)$ yields $\left.\nabla_{x} \Phi(x) c(x)\right|_{x=\Phi^{-1}(z)}=$

$$
\begin{aligned}
& \left(\begin{array}{c}
\left(S\left(\Phi^{-1}(\xi, \eta)\right)-R\right) \xi \\
0
\end{array}\right)=H(\xi, \eta) \text {. Therefore } \\
& \dot{z}=\left(\begin{array}{c}
\dot{\xi} \\
\dot{\eta}
\end{array}\right)=B(\xi, \eta)+H(\xi, \eta)=\left(\begin{array}{c}
S\left(\Phi^{-1}(\xi, \eta)\right) \xi \\
q(\xi, \eta)
\end{array}\right) .
\end{aligned}
$$

Defining $p_{i}(\xi, \eta)=\nabla_{\Phi^{-1}(\xi, \eta)} \psi\left(\Phi^{-1}(\xi, \eta)\right) s_{i}\left(\Phi^{-1}(\xi, \eta)\right)$, then the system (44) is equal to the system $(21)$ with $k_{i}(\xi, \eta)$ given in (36). By assumption, the positive definite function $\left.W(\psi(x))\right|_{x=\Phi^{-1}(\xi, \eta)}=W(\eta)$ satisfies

$\frac{\partial W(\psi(x))}{\partial \psi(x)} \frac{\partial \psi(x)}{\partial x}\left(\widehat{f}(x)-\sum_{i=1}^{r} s_{i}(x) L_{f}^{i-1} h(x)\right) \leq \gamma W(\psi(x))+\gamma_{0}$,

which can be rewritten in the coordinates $(\xi, \eta)$ as in (35) while (42) can be rewritten as (32). Therefore, by Lemma 3 , the claims follow.

Remark 11. With arguments similar to those in Theorem 3, the claims of Theorem 4 can be restated in a local fashion.

\section{Discretization SChemes}

Since for a numerical implementation of any integration method a discretization scheme is required, in this section the problem of discretization of system (21) is addressed. In fact, note that the solution trajectory of the difference equations resulting from the discretization of (21) may diverge even though the trajectories of the underlying ODEs converge to the solution manifold. Hence we now propose two integration schemes for system (21) which preserve the properties discussed in Section III. The advantage of the underlying schemes is that the stabilization term need not to be discretized by the same method applied to the constrained system. In the remainder of the paper we use the notation $x:=x(t)$ and $x^{+}:=x(t+T)$, where $t=\widetilde{k} T, \forall \widetilde{k} \in \mathbb{N}$ and $T \in \mathbb{R}_{>0}$ is the sampling period.

Consider the discrete-time system

$$
\frac{\xi^{+}-\xi}{T}=K(\xi, \eta) \xi, \frac{\eta^{+}-\eta}{T}=\varphi_{T}\left(q_{0}\right)+Q(\xi, \eta) \xi,
$$

with $\eta(t) \in \mathbb{R}^{n-r}, \xi(t) \in \mathbb{R}^{r}, Q: \mathbb{R}^{r} \times \mathbb{R}^{n-r} \rightarrow \mathbb{R}^{n-r \times r}, Q=$ $\left[q_{1}, \ldots, q_{r}\right], \varphi_{T}: \mathbb{R}^{n-r} \rightarrow \mathbb{R}^{n-r}, q_{0}$ and $q_{i}$, for $i=1, \ldots, r$ defined in (20), and $K: \mathbb{R}^{r} \times \mathbb{R}^{n-r} \rightarrow \mathbb{R}^{r \times r}$. Recalling the definition of $k_{i}$ given in (22), let $K(\xi, \eta)$ be such that

$$
\begin{aligned}
K(\xi, \eta) & =-\frac{\delta^{2}}{2} \operatorname{diag}\left(q_{1}^{2}(\xi, \eta), \ldots, q_{r}^{2}(\xi, \eta)\right)-\epsilon I= \\
& =\operatorname{diag}\left(k_{1}, \ldots, k_{r}\right) .
\end{aligned}
$$

Observe that, for $T \rightarrow 0$, system (45) is equal to system (21), provided the map $\varphi_{T}\left(q_{0}\right)$ is such that

$$
\lim _{T \rightarrow 0} \varphi_{T}\left(q_{0}\right)=q_{0} .
$$

For instance, if

$$
\varphi_{T}\left(q_{0}\right)=q_{0},
$$

then system (45)-(48) represents the forward Euler discretization of system (21). Similarly to Theorem 2 for the continuoustime system (21), for the discrete-time system (45) the following result holds.

Theorem 5. Consider system (45) and let $\widetilde{U} \subseteq \mathbb{R}^{n}$ be a compact set which contains the origin. For all $T$ such that

$$
0<T<\frac{2}{\max _{i=1, \ldots, r}\left(\max _{(\xi, \eta) \in \widetilde{U}}\left(-k_{i}(\xi, \eta)\right)\right)}
$$

there exists a neighborhood of the origin $U \subseteq \widetilde{U}$ such that the following claims hold.

1) $\xi(t)$ and $\eta(t)$ exist for all $\xi(0) \in \mathbb{R}^{r}$ and $\eta(0) \in \mathbb{R}^{n-r}$;

2) $\lim _{t \rightarrow+\infty} \xi(t)=0$, for all $(\xi(0), \eta(0)) \in U$.

Proof. The proof of claim 1) trivially follows from the fact that the right hand side of equation (45) is continuous. To prove claim 2) consider the positive definite and radially unbounded function $V(\xi)=\xi^{\top} \xi$. It follows that

$\frac{\Delta V}{T}=\frac{V\left(\xi^{+}\right)-V(\xi)}{T}=\xi^{\top} \frac{\left((I+T K(\xi, \eta))^{\top}(I+T K(\xi, \eta))-I\right)}{T} \xi=$ $=\xi^{\top}\left(T K(\xi, \eta)^{\top} K(\xi, \eta)+2 K(\xi, \eta)\right) \xi=\xi^{\top} D_{T}(\xi, \eta) \xi$,

where

$D_{T}(\xi, \eta)=T K(\xi, \eta)^{\top} K(\xi, \eta)+2 K(\xi, \eta)=\operatorname{diag}\left(d_{1}, \ldots, d_{r}\right)$, 
with

$$
d_{i}=T k_{i}(\xi, \eta)^{2}+2 k_{i}(\xi, \eta)=\left(T k_{i}(\xi, \eta)+2\right) k_{i}(\xi, \eta)
$$

for $i=1, \ldots, r$. Note now that the selection of $T$ as in (49) implies that $-2<T k_{i}(\xi, \eta)<0$ and, since $k_{i}(\xi, \eta)<0$, also $d_{i}<0$ for $i=1, \ldots, r$. Hence, $\Delta V=T \sum_{i=1}^{r} d_{i} \xi_{i}^{2}<0$, for all $\xi \neq 0$, proving claim 2 ).

Note that different discretization schemes yield different properties. For instance, consider the modified discrete-time system

$$
\frac{\xi^{+}-\xi}{T}=K(\xi, \eta) \xi^{+}, \frac{\eta^{+}-\eta}{T}=\varphi_{T}\left(q_{0}\right)+Q(\xi, \eta) \xi .
$$

Observe that, since $\lim _{T \rightarrow 0} \xi^{+}=\lim _{T \rightarrow 0} \xi(t+T)=\xi(t)$ and (47) holds, system (52) is equal to system (21) when $T \rightarrow 0$. Hence, the following result holds.

Theorem 6. Consider system (52). Then the following claims hold for any $T>0$.

1) $\xi(t)$ and $\eta(t)$ exist for all $\xi(0) \in \mathbb{R}^{r}$ and $\eta(0) \in \mathbb{R}^{n-r}$;

2) $\lim _{t \rightarrow+\infty} \xi(t)=0$ for all $\xi(0) \in \mathbb{R}^{r}$ and $\eta(0) \in \mathbb{R}^{n-r}$.

Proof. To prove claim 1) consider the explicit representation of system (52), namely

$$
\begin{aligned}
& \frac{\xi^{+}-\xi}{T}=(I-T K(\xi, \eta))^{-1} K(\xi, \eta) \xi, \\
& \frac{\eta^{+}-\eta}{T}=\varphi_{T}\left(q_{0}\right)+Q(\xi, \eta) \xi
\end{aligned}
$$

and note that the right hand side of (53) is continuous, proving the claim. The proof of claim 2) follows the same steps of the proof of Theorem 5 with the difference that $D_{T}$ now has the expression

$$
\begin{aligned}
D_{T}(\xi, \eta) & =\frac{(I-T K(\xi, \eta))^{-\top}(I-T K(\xi, \eta))^{-1}-I}{T}= \\
& =\operatorname{diag}\left(d_{1}, \ldots, d_{r}\right)
\end{aligned}
$$

with

$$
d_{i}=\frac{1}{T\left(1-T k_{i}(\xi, \eta)\right)^{2}}-\frac{1}{T}=\frac{2-T k_{i}(\xi, \eta)}{\left(1-T k_{i}(\xi, \eta)\right)^{2}} k_{i}(\xi, \eta)
$$

for $i=1, \ldots, r$. Note now that since $k_{i}(\xi, \eta)<0$ also $d_{i}<0$, for $i=1, \ldots, r$. Hence $D_{T}(\xi, \eta)<0$, proving claim 2).

Theorem 6 ensures that the manifold (6) is globally attractive and that the solutions of the system exist for any $T>0$. We consider now the stability properties of the zero equilibrium of systems (45) and (52). The following result provides a discrete-time equivalent of Theorem 3.

Theorem 7. Let the assumptions of Theorem 3 hold. Let $\widetilde{U} \subseteq \mathbb{R}^{\bar{n}} \times B$ be a closed set which contains the origin. Let $\overline{\bar{T}}$ be such that

$$
\frac{1}{n-r} \sum_{i=1}^{n-r} \frac{W\left(\eta^{+}\right)-W(\eta)}{\eta_{i}^{+}-\eta_{i}} \varphi_{T_{i}}(\eta) \leq \gamma W(\eta)
$$

in which $\varphi_{T_{i}}$ represents the $i$-th entries of the vector $\varphi_{T}$, and

$$
\max _{(\xi, \eta) \in \widetilde{U}} \frac{\left(\frac{1}{n-r} \sum_{i=1}^{n-r} \frac{W\left(\eta^{+}\right)-W(\eta)}{\eta_{i}^{+}-\eta_{i}}\right)^{2}}{W(\eta)} \leq \bar{W},
$$

for all $0<T \leq \overline{\bar{T}}$. Let $T \leq \min \left(\frac{\bar{T}}{2}, \overline{\bar{T}}\right)$ if the discretization (45) is used and $T \leq \min \left(\frac{\sqrt{5}-1}{4} \bar{T}, \overline{\bar{T}}\right)$ if the discretization (52) is used, where $\bar{T}$ is such that (49) holds. Then for this selection of $T$ and for $\delta$ and $\epsilon$ selected as in Theorem 3, there exists a neighborhood of the origin $U \subseteq \widetilde{U}$ such that the following claims hold.

1) $\xi(t)$ and $\eta(t)$ exist, for all $(\xi(0), \eta(0)) \in U$;

2) $\lim _{t \rightarrow+\infty} \xi(t)=0$, for all $(\xi(0), \eta(0)) \in U$;

3) $\lim _{t \rightarrow+\infty} \eta(t)=0$, for all $(\xi(0), \eta(0)) \in U$.

Proof. Consider first the discretization scheme (45). The proof of claims 1) and 2) trivially follow from Theorem 5. To prove claim 3), consider the positive definite function $V(\xi, \eta)=$ $W(\eta)+\xi^{\top} \xi$. The variation of $V(\xi, \eta)$ along the trajectories of the system in a time interval $T$ is

$$
\begin{aligned}
& \frac{\Delta V}{T}=\frac{W\left(\eta^{+}\right)-W(\eta)}{T}+\frac{\xi^{+\top} \xi^{+}-\xi^{\top} \xi}{T}=\frac{1}{n-r} \times \\
& \times \sum_{i=1}^{n-r} \frac{W\left(\eta^{+}\right)-W(\eta)}{\eta_{i}^{+}-\eta_{i}} \frac{\eta_{i}^{+}-\eta_{i}}{T}+\xi^{\top} D_{T}(\xi, \eta) \xi=\frac{1}{n-r} \times \\
& \times \sum_{i=1}^{n-r} \frac{W\left(\eta^{+}\right)-W(\eta)}{\eta_{i}^{+}-\eta_{i}}\left(\varphi_{T_{i}}(\eta)+Q_{i}(\xi, \eta) \xi\right)+\xi^{\top} D_{T}(\xi, \eta) \xi
\end{aligned}
$$

where $D_{T}(\xi, \eta)$ is defined in (50) and $Q_{i}(\xi, \eta)$ is the $i$-th row of the matrix $Q(\xi, \eta)$. Note now that

$$
\begin{aligned}
& \sum_{i=1}^{n-r} \frac{W\left(\eta^{+}\right)-W(\eta)}{\eta_{i}^{+}-\eta_{i}} Q_{i}(\xi, \eta) \xi= \\
& =\left[\frac{W\left(\eta^{+}\right)-W(\eta)}{\eta_{1}^{+}-\eta_{1}} \ldots \frac{W\left(\eta^{+}\right)-W(\eta)}{\eta_{n-r}^{+}-\eta_{n-r}}\right] \sum_{i=1}^{r} q_{i} \xi_{i} .
\end{aligned}
$$

By replacing the previous equation in (58) and by applying Young inequality yields

$$
\begin{aligned}
& \frac{\Delta V}{T} \leq \frac{1}{n-r} \sum_{i=1}^{n-r} \frac{W\left(\eta^{+}\right)-W(\eta)}{\eta_{i}^{+}-\eta_{i}} \varphi_{T_{i}}(\eta)+\frac{1}{(n-r)^{2}} \sum_{i=1}^{n-r} \frac{1}{2 \delta^{2}} \times \\
& \times\left(\frac{W\left(\eta^{+}\right)-W(\eta)}{\eta_{i}^{+}-\eta_{i}}\right)^{2}+\sum_{i=1}^{r} \frac{\delta^{2}}{2}\left\|q_{i}(\xi, \eta)\right\|^{2} \xi^{\top} \xi+\xi^{\top} D_{T}(\xi, \eta) \xi,
\end{aligned}
$$

hence

$\frac{\Delta V}{T} \leq \frac{1}{n-r} \sum_{i=1}^{n-r} \frac{W\left(\eta^{+}\right)-W(\eta)}{\eta_{i}^{+}-\eta_{i}} \varphi_{T_{i}}(\eta)+\frac{1}{(n-r)^{2}} \sum_{i=1}^{n-r} \frac{1}{2 \delta^{2}} \times$
$\times\left(\frac{W\left(\eta^{+}\right)-W(\eta)}{\eta_{i}^{+}-\eta_{i}}\right)^{2}+\sum_{i=1}^{n-r}\left(T k_{i}(\xi, \eta)^{2}+2 k_{i}(\xi, \eta)-k_{i}(\xi, \eta)-\epsilon\right) \xi_{i}^{2}$,

where the last inequality is obtained by replacing the expression of (51) in $D_{T}(\xi, \eta)$. Since $T \leq \frac{\bar{T}}{2}$, with $\bar{T}$ satisfying (49), then 
$-1<T k_{i}(\xi, \eta)<0$ and, as a consequence

$$
\begin{aligned}
& \frac{\Delta V}{T} \leq \frac{1}{n-r} \sum_{i=1}^{n-r} \frac{W\left(\eta^{+}\right)-W(\eta)}{\eta_{i}^{+}-\eta_{i}} \varphi_{T_{i}}(\eta)+ \\
& +\frac{1}{(n-r)^{2}} \sum_{i=1}^{n-r} \frac{1}{2 \delta^{2}}\left(\frac{W\left(\eta^{+}\right)-W(\eta)}{\eta_{i}^{+}-\eta_{i}}\right)^{2}-\epsilon \sum_{i=1}^{n-r} \xi_{i}^{2} .
\end{aligned}
$$

Note that, under assumption (57), and by selecting $\delta \geq \bar{\delta}$ where $\bar{\delta}$ is given in (29), for some $0<\beta<-\gamma$, yields

$$
\frac{\Delta V}{T} \leq \frac{1}{n-r} \sum_{i=1}^{n-r} \frac{W\left(\eta^{+}\right)-W(\eta)}{\eta_{i}^{+}-\eta_{i}} \varphi_{T_{i}}(\eta)+\beta W(\eta)-\epsilon \sum_{i=1}^{n-r} \xi_{i}^{2},
$$

and, under assumption (56) and by selecting $\epsilon \geq \bar{\epsilon}$ where $\bar{\epsilon}=-\gamma-\beta$ yields $\frac{\Delta V}{T} \leq(\gamma+\beta) W(\eta)-\epsilon \sum_{i=1}^{n-r} \xi_{i}^{2} \leq$ $(\gamma+\beta)\left(W(\eta)+\xi^{\top} \xi\right)=(\gamma+\beta) V(\xi, \eta)$. Hence $\Delta V \leq$ $T(\gamma+\beta) V(\xi, \eta)<0$, for all $(\xi, \eta) \neq(0,0)$, proving claim 3$)$. Consider now the discretization (52). Claims 1) and 2) follow from Theorem 6. The proof of claim 3) follows the same steps of the previous proof with the difference that $D_{T}$ has the expression in (54)-(55). Hence the inequality (59) becomes

$$
\begin{aligned}
& \frac{\Delta V}{T} \leq \frac{1}{n-r} \sum_{i=1}^{n-r} \frac{W\left(\eta^{+}\right)-W(\eta)}{\eta_{i}^{+}-\eta_{i}} \varphi_{T_{i}}(\eta)+\frac{1}{(n-r)^{2}} \sum_{i=1}^{n-r} \frac{1}{2 \delta^{2}} \times \\
& \times\left(\frac{W\left(\eta^{+}\right)-W(\eta)}{\eta_{i}^{+}-\eta_{i}}\right)^{2}+\sum_{i=1}^{n-r}\left(\left(\frac{2-T k_{i}(\xi, \eta)}{\left(1-T k_{i}(\xi, \eta)\right)^{2}}-1\right) k_{i}(\xi, \eta)-\epsilon\right) \xi_{i}^{2} .
\end{aligned}
$$

By means of simple calculations it is easy to show that the inequality

$$
\sum_{i=1}^{n-r}\left(\frac{2-T k_{i}(\xi, \eta)}{\left(1-T k_{i}(\xi, \eta)\right)^{2}}-1\right) k_{i}(\xi, \eta)<0
$$

holds for $\left(\frac{1-\sqrt{5}}{2}\right)<T k_{i}(\xi, \eta)<0$, which is clearly satisfied for $T \leq \frac{\sqrt{5}-1}{4} \bar{T}$. Therefore the claim follows by applying the same steps in equations (60) and (61).

Remark 12. For $T \rightarrow 0$ equation (56) becomes

$$
\lim _{T \rightarrow 0} \frac{1}{n-r} \sum_{i=1}^{n-r} \frac{W(\eta(t+T))-W(\eta(t))}{\eta_{i}(t+T)-\eta_{i}(t)} \varphi_{T_{i}}(\eta)=\lim _{T \rightarrow 0} \frac{\Delta W}{T} \leq \gamma W .
$$

Hence condition (56) is the discrete-time counterpart of (25) with $\gamma<0$ and $\gamma_{0}=0$. Similarly, condition (57) is the discretetime counterpart of (32). This observation implies that for $T$ sufficiently small, (25) and (32) imply (56) and (57).

Remark 13. While the discretization scheme in (52) ensures global attractivity of the solution manifold, for the discretization scheme (45) attractivity holds only locally unless $q_{i}$, for $i=$ $1, \ldots, r$ are bounded, see Theorems 5 and 6 . However, this comes at the cost of a smaller sampling time required to preserve the stability properties of the equilibrium points, see the assumptions of Theorem 7.

Remark 14. For the general case in which $m>1$, the mappings $K(\xi, \eta)$ and $Q(\xi, \eta)$ in (45)-(52) can be written as $K(\xi, \eta)=\operatorname{diag}\left(k_{1}^{1}(\xi, \eta), \ldots, k_{r_{m}}^{m}(\xi, \eta)\right)$ and $Q(\xi, \eta)=$ $\left[q_{1}^{1}, \ldots, q_{r_{m}}^{m}\right]$, respectively, where $k_{i}^{j}(\xi, \eta)$ is given in equation (34). Observe also that the claims of Theorems 5, 6 and 7 remain valid whenever $k_{i}(\xi, \eta)$ in (49) is replaced by $k_{i}^{j}(\xi, \eta)$, for $j=1, \ldots, m$. The proof follows the same steps as the case $m=1$, in which $d_{i}, q_{i}$ and $r$ are replaced by $d_{i}^{j}, q_{i}^{j}$ and $r_{j}$, respectively.

Remark 15. By using the concepts of relative degree and zero dynamics for sampled nonlinear systems (see [51] and [52]), and with similar arguments as those in Lemma 3 and in Theorem 4, the claims of Theorems 5, 6 and 7 can be restated in the original coordinates system.

The results presented in Sections II, III and IV outline a procedure to simulate DAE systems, as described in the following algorithm.

\section{Algorithm \\ Data: \\ - $f, g$ and $h$ as given in (1) and such that the index $\nu$ is well-defined; \\ - $x_{0}=x(0) \in \mathbb{R}^{n}$ and a function $W$ such that assumptions of Theorem 3 holds.}

Output: $x^{+}$such that

- Claims 1) and 2) of Theorem 5 hold if the discretization scheme (45) is used;

- Claims 1) and 2) of Theorem 6 hold if the discretization scheme (52) is used;

- Claims 1), 2) and 3) of Theorem 7 hold if $x^{\circ}$ is locally asymptotically stable.

Step 1. Set $\phi_{i}(x)=L_{f}^{i-1} h(x)$ for $i=1, \ldots, r$ and complete the set of $\phi_{i}(x)$ for $i=r+1, \ldots, n$ such that the Jacobian of $\Phi(x)$ is nonsingular.

Step 2. Write the system in the coordinates $(\xi, \eta)=\Phi(x)$ and compute the mappings $q_{i}$, for $i=1, \ldots, r$, such that $\dot{\eta}=q(\xi, \eta)=q_{0}(\eta)+\sum_{i=1}^{r} q_{i}(\xi, \eta) \xi_{i}$.

Step 3. Compute the parameters $\gamma, \gamma_{0}$ and $\bar{W}$ which satisfy (25) and (26).

Step 4. IF $\gamma_{0}=0$ and $\gamma<0$ in Step 3 THEN Select $\epsilon \geq-\gamma-\beta$ and $\delta<\bar{\delta}=\frac{1}{2 \beta} \bar{W}$, with $\beta \in[-\gamma, 0]$.

ELSE select $\epsilon>0$ and $\delta$ such that the inequality $\delta<\bar{\delta}=$ $\frac{1}{2 \beta} \bar{W}$ holds for some $\beta>0$.

Step 5. Compute $\bar{T}$ such that (49) holds and $\overline{\bar{T}}$ such that (56) and (57) hold.

Step 6. IF $\gamma_{0}=0$ and $\gamma<0$ in Step 3 THEN Select a sampling time $T \leq \min \left(\frac{\bar{T}}{2}, \overline{\bar{T}}\right)$ OR $T \leq \min \left(\frac{\sqrt{5}-1}{4} \bar{T}, \overline{\bar{T}}\right)$ ELSE select $T \leq \bar{T}$ OR $T>0$.

Step 7. Accordingly to the choices in Step 6, advance the solution from the approximate state $(\xi, \eta)$ to the approximate state $\left(\xi^{+}, \eta^{+}\right)$according to scheme (45) $\mathbf{O R}$ according to scheme (52), in which $\varphi_{T}(q)$ is any one-step scheme such that (47) holds.

Step 8. Set $x^{+}=\Phi\left(\xi^{+}, \eta^{+}\right)^{-1}$. 


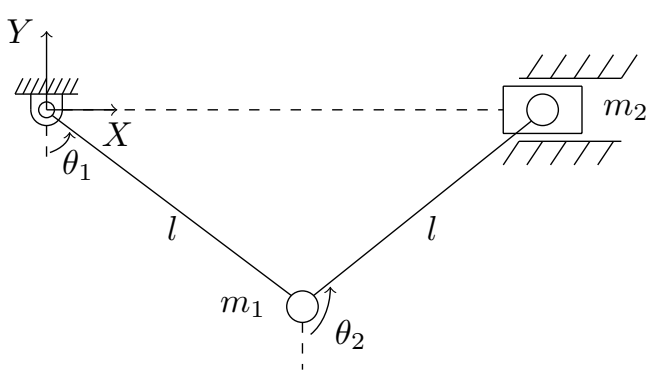

Fig. 1: A slider-crank mechanism.

\section{EXAMPLE: A SLIDER-CRANK MECHANISM}

Consider the slider-crank mechanism in Fig. 1. Let $\theta=$ $\left(\theta_{1}, \theta_{2}\right)^{\top}$ denotes the angular positions of the two masses $m_{1}$ and $m_{2}$, respectively, with respect to the vertical axis and let the rods have equal length $l$. In addition, viscous friction with coefficient $c$ acts on the mass $m_{1}$ and gravity force, where $g_{v}$ is the gravitational acceleration, acts on both $m_{1}$ and $m_{2}$. The motion of the system is subject to a holonomic constraint which constrains the mass $m_{2}$ to slide horizontally. The kinetic and potential energy in terms of $\theta, \dot{\theta}$ are

$$
\begin{aligned}
T(\theta, \dot{\theta}) & =\frac{1}{2}\left(m_{1}+m_{2}\right) l^{2} \dot{\theta}_{1}^{2}+\frac{1}{2} m_{2} l^{2}\left(\dot{\theta}_{2}^{2}+2 \dot{\theta}_{1} \dot{\theta}_{2}\right) \cos \left(\theta_{2}-\theta_{1}\right), \\
U(\theta) & =\left(m_{1}+m_{2}\right) g_{v} l\left(1-\cos \left(\theta_{1}\right)\right)-m_{2} g_{v} l \cos \left(\theta_{2}\right) .
\end{aligned}
$$

The application of the Euler equations yields the equations of motion

$$
\begin{aligned}
& 0=h(q), \quad \dot{q}=v, \\
& \dot{v}=M^{-1}(q) \bar{f}(q, v)-M^{-1}(q) G^{\top}(q) \lambda,
\end{aligned}
$$

where $q=\theta, v=\dot{\theta}$ and

$$
\begin{aligned}
M(\theta) & =\left[\begin{array}{cc}
\left(m_{1}+m_{2}\right) l^{2} & m_{2} l^{2} \cos \left(\theta_{2}-\theta_{1}\right) \\
m_{2} l^{2} \cos \left(\theta_{2}-\theta_{1}\right) & m_{2} l^{2}
\end{array}\right], \\
\bar{f}(\theta, \dot{\theta}) & =\left[\begin{array}{cc}
m_{2} l^{2} \sin \left(\theta_{2}-\theta_{1}\right) \dot{\theta}_{2}^{2}-c \dot{\theta}_{1}-\left(m_{1}+m_{2}\right) l g_{v} \sin \left(\theta_{1}\right) \\
-m_{2} l^{2} \sin \left(\theta_{2}-\theta_{1}\right) \dot{\theta}_{1}^{2}-m_{2} l g_{v} \sin \left(\theta_{2}\right)
\end{array}\right], \\
h(\theta) & =\cos \left(\theta_{1}\right)+\cos \left(\theta_{2}\right), \\
G(\theta) & =\frac{\partial h(\theta)}{\partial \theta}=\left[\begin{array}{ll}
-\sin \left(\theta_{1}\right) & -\sin \left(\theta_{2}\right)
\end{array}\right]^{\top} .
\end{aligned}
$$

Assume, for simplicity of notation, that the parameters $m_{1}$, $m_{2}$ and $l$ have unit value. Equations (62)-(63) can be rewritten in the state space form (1), with state $x=\left[\begin{array}{llll}x_{1} & x_{2} & x_{3} & x_{4}\end{array}\right]^{\top}=$ $\left[\begin{array}{llll}\theta_{1} & \theta_{2} & \dot{\theta}_{1} & \dot{\theta}_{2}\end{array}\right]^{\top}, f=\left[\begin{array}{llll}f_{1} & f_{2} & f_{3} & f_{4}\end{array}\right]^{\top}, g=\left[\begin{array}{llll}g_{1} & g_{2} & g_{3} & g_{4}\end{array}\right]^{\top}$,

$$
\begin{aligned}
f_{1}(x)= & x_{3}, \quad f_{2}(x)=x_{4}, \\
f_{3}(x)= & \frac{\sin \left(x_{1}-x_{2}\right) x_{4}{ }^{2}+2 g_{v} \sin \left(x_{1}\right)+c x_{3}}{\left(\cos \left(x_{1}-x_{2}\right)\right)^{2}-2}+ \\
& -\frac{\cos \left(x_{1}-x_{2}\right)\left(-\sin \left(x_{1}-x_{2}\right) x_{3}{ }^{2}+g_{v} \sin \left(x_{2}\right)\right)}{\left(\cos \left(x_{1}-x_{2}\right)\right)^{2}-2}, \\
f_{4}(x)= & \frac{-2 \sin \left(x_{1}-x_{2}\right) x_{3}{ }^{2}+2 g_{v} \sin \left(x_{2}\right)}{\left(\cos \left(x_{1}-x_{2}\right)\right)^{2}-2}+ \\
& -\frac{\cos \left(x_{1}-x_{2}\right)\left(\sin \left(x_{1}-x_{2}\right) x_{4}{ }^{2}+2 g_{v} \sin \left(x_{1}\right)+c x_{3}\right)}{\left(\cos \left(x_{1}-x_{2}\right)\right)^{2}-2},
\end{aligned}
$$

$$
\begin{aligned}
& g_{1}(x)=0, \quad g_{2}(x)=0, \\
& g_{3}(x)=\frac{\sin \left(x_{1}\right)}{\left(\cos \left(x_{1}-x_{2}\right)\right)^{2}-2}-\frac{\cos \left(x_{1}-x_{2}\right) \sin \left(x_{2}\right)}{\left(\cos \left(x_{1}-x_{2}\right)\right)^{2}-2}, \\
& g_{4}(x)=-\frac{\cos \left(x_{1}-x_{2}\right) \sin \left(x_{1}\right)}{\left(\cos \left(x_{1}-x_{2}\right)\right)^{2}-2}+2 \frac{\sin \left(x_{2}\right)}{\left(\cos \left(x_{1}-x_{2}\right)\right)^{2}-2},
\end{aligned}
$$

and

$$
h(x)=\cos \left(x_{1}\right)+\cos \left(x_{2}\right) .
$$

Note that for $x_{1} \in(-\pi, \pi]$ and $x_{2} \in(-\pi, \pi]$ this system has two equilibrium points, namely $x_{1}^{\circ}=(\pi, 0,0,0)$ and $x_{2}^{\circ}=$ $(0, \pi, 0,0)$, respectively.

Remark 16. The relative degree of system (1)-(64)-(65) is not well-defined at $x_{2}^{\circ}$ as $L_{g} L_{f} h\left(x_{2}^{\circ}\right)=0$. Observe that this issue is due to the existence of multiple solutions for the constraint equation $h\left(x_{2}^{\circ}\right)=0$, namely the ones in which the two rods move in the same or opposite directions respectively. Note however that the constraint (66) can be rewritten as

$$
h(x)=2 \cos \left(\frac{x_{1}+x_{2}}{2}\right) \cos \left(\frac{x_{1}-x_{2}}{2}\right)=0,
$$

the solutions of which satisfy

$$
\pm\left((1+2 j) \pi-x_{1}\right)-x_{2}=0,
$$

for $j \in \mathbb{N}$. The singularity in the definition of the relative degree can be avoided, for instance, by replacing equation (67) with

$$
h(x)=x_{1}+x_{2}-\pi=0,
$$

i.e. equation (68) with $j=0$, which selects the particular solution in which the rods move in opposite directions.

Although the following analysis can be carried out for any solution of (68) we focus on the constraint (69). Note that, in this case, the matrix $G(\theta)$ in equations (63) becomes $G(\theta)=$ $\left[\begin{array}{ll}1 & 1\end{array}\right]^{\top}$, while $g$ in (65) simplifies to

$$
\begin{aligned}
& g_{1}(x)=0, \quad g_{2}(x)=0, \\
& g_{3}(x)=-\frac{1}{\left(\cos \left(x_{1}-x_{2}\right)\right)^{2}-2}+\frac{\cos \left(x_{1}-x_{2}\right)}{\left(\cos \left(x_{1}-x_{2}\right)\right)^{2}-2}, \\
& g_{4}(x)=\frac{\cos \left(x_{1}-x_{2}\right)}{\left(\cos \left(x_{1}-x_{2}\right)\right)^{2}-2}-\frac{2}{\left(\cos \left(x_{1}-x_{2}\right)\right)^{2}-2} .
\end{aligned}
$$

Since the relative degree of system (1)-(64)-(70) at $x_{2}^{\circ}$ is $r=2$, there exists a coordinate transformation which transforms the system in the normal form (5). For instance, the mapping $\left[\begin{array}{llll}\xi_{1} & \xi_{2} & \eta_{1} & \eta_{2}\end{array}\right]^{\top}=\left[\begin{array}{llll}x_{1}+x_{2}-\pi & x_{3}+x_{4} & x_{1} & x_{3}\end{array}\right]^{\top}$, calculated according to Proposition 1, is such that

$$
q(\xi, \eta)=\left[\begin{array}{c}
\eta_{2} \\
\frac{\left(-2 \eta_{2}{ }^{2}+2 \eta_{2} \xi_{2}-\xi_{2}{ }^{2}\right) \sin \left(-2 \eta_{1}+\xi_{1}\right)-c \eta_{2}}{2 \cos \left(-2 \eta_{1}+\xi_{1}\right)+3}+ \\
+\frac{-2 g_{v} \sin \left(\eta_{1}\right)-g_{v} \sin \left(\xi_{1}-\eta_{1}\right)}{2 \cos \left(-2 \eta_{1}+\xi_{1}\right)+3}
\end{array}\right],
$$

in equations (5) or, equivalently $q(\xi, \eta)=q_{0}(\eta)+q_{1}(\xi, \eta) \xi_{1}+$ $q_{2}(\xi, \eta) \xi_{2}$, where

$$
q_{0}(\eta)=\left[\begin{array}{c}
\eta_{2} \\
\frac{2 \eta_{2}^{2} \sin \left(2 \eta_{1}\right)-c \eta_{2}-g_{v} \sin \left(\eta_{1}\right)}{2 \cos \left(2 \eta_{1}\right)+3}
\end{array}\right],
$$




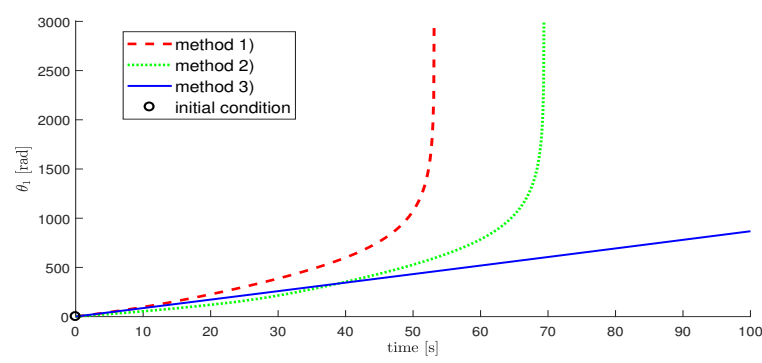

(a)

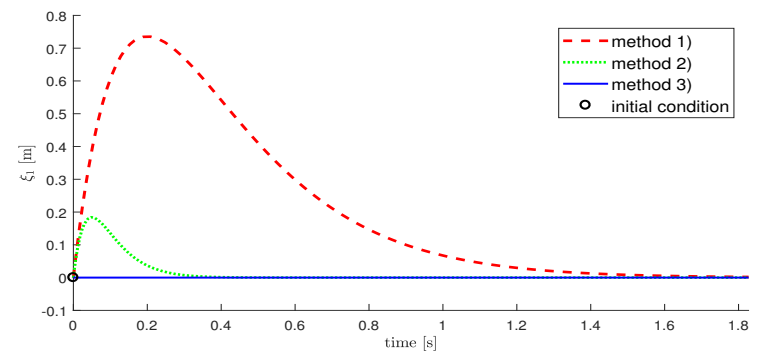

(c)

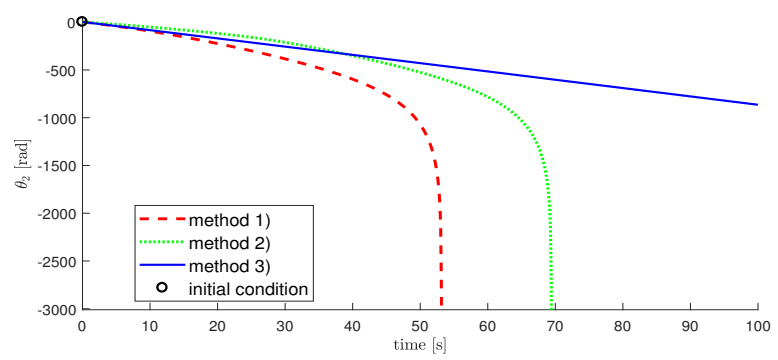

(b)

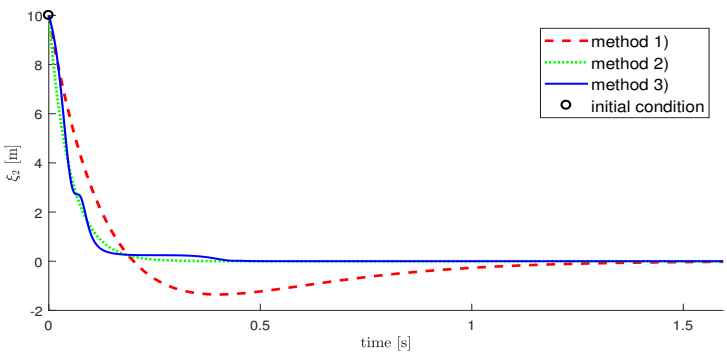

(d)

Fig. 2: Comparison of three stabilization methods (method 1 in dashed-red line, method 2 in dotted-green line and method 3 in solid-blue line) for the same initial condition (black circle): (a) time histories of $\theta_{1}$; (b) time histories of $\theta_{2}$; (c) time histories of $\xi_{1}$; (d) time histories of $\xi_{2}$.

$$
q_{1}(\xi, \eta)=\left[\begin{array}{c}
0 \\
\frac{1}{\xi_{1}}\left(\frac{-2 \sin \left(\xi_{1}-2 \eta_{1}\right) \eta_{2}{ }^{2}-2 g_{v} \sin \left(\eta_{1}\right)-g_{v} \sin \left(\xi_{1}-\eta_{1}\right)-c \eta_{2}}{2 \cos \left(-2 \eta_{1}+\xi_{1}\right)+3}+\right. \\
\left.-\frac{2 \eta_{2}{ }^{2} \sin \left(2 \eta_{1}\right)-c \eta_{2}-g_{v} \sin \left(\eta_{1}\right)}{2 \cos \left(2 \eta_{1}\right)+3}\right)
\end{array}\right]
$$

and

$$
q_{2}(\xi, \eta)=\left[\begin{array}{c}
0 \\
-\frac{\sin \left(-2 \eta_{1}+\xi_{1}\right) \xi_{2}}{2 \cos \left(-2 \eta_{1}+\xi_{1}\right)+3}+2 \frac{\eta_{2} \sin \left(-2 \eta_{1}+\xi_{1}\right)}{2 \cos \left(-2 \eta_{1}+\xi_{1}\right)+3}
\end{array}\right] .
$$

Accordingly, the zero dynamic of the system (1)-(64)-(70) is

$$
\dot{\eta}_{1}=\eta_{2}, \quad \dot{\eta}_{2}=\frac{4 \eta_{2}^{2} \sin \left(\eta_{1}\right) \cos \left(\eta_{1}\right)-g \sin \left(\eta_{1}\right)-c \eta_{2}}{2 \cos \left(2 \eta_{1}\right)+3} \text {. }
$$

It is easy to show that the equilibrium $\left(\eta_{1}^{\circ}, \eta_{2}^{\circ}\right)=(0,0)$ is stable for $c \geq 0$ and locally asymptotically stable for any $c>0$. For instance, let $\mathcal{M}$ be as in (6), then the function

$$
\begin{aligned}
E^{*} & =\left.T\left(\Phi(\xi, \eta)^{-1}\right)\right|_{\mathcal{M}}+\left.U\left(\Phi(\xi, \eta)^{-1}\right)\right|_{\mathcal{M}}-g_{v}= \\
& =2 \eta_{2}^{2}\left(\cos \left(\eta_{1}\right)\right)^{2}-g_{v} \cos \left(\eta_{1}\right)+\frac{1}{2} \eta_{2}{ }^{2}+g_{v}
\end{aligned}
$$

is positive definite in a neighborhood $\mathcal{B} \subseteq \mathbb{R}^{2}$ of the origin and such that $E^{*}(0)=0$. Moreover, the time derivative of $E^{*}$ along the trajectories of the zero dynamics $(72)$ is $\dot{E}^{*}=$ $-c \eta_{2}^{2} \leq 0$, which proves that the equilibrium $\left(\eta_{1}^{\circ}, \eta_{2}^{\circ}\right)$ is stable for $c \geq 0$. In the case $c>0$, note that $\dot{E}^{*}=0$ implies $\eta_{2}=0$ which replaced in equations (72) yields $\eta_{1}=j \pi$ for $j \in$ $\mathbb{N}_{\geq}$. Hence the set $\left\{\left(\eta_{1}, \eta_{2}\right): \dot{E}^{*}\left(\eta_{1}, \eta_{2}\right)=0\right\} \cap \mathcal{B}$ does not contain any trajectory besides the trivial one $\left(\eta_{1}, \eta_{2}\right)=(0,0)$, thus asymptotic stability of $\left(\eta_{1}^{\circ}, \eta_{2}^{\circ}\right)$ follows from LaSalle's invariance principle [53], which proves the claim. In addition, since the equilibrium $\left(\eta_{1}^{\circ}, \eta_{2}^{\circ}\right)$ is the restriction of $x_{2}^{\circ}$ on the solution manifold $\mathcal{M}$ then, by Theorem 1 , these have the same stability properties. Note that by studying the stability properties of the equilibrium point $\left(\eta_{1}^{\circ}, \eta_{2}^{\circ}\right)=(\pi, 0)$ of the zero dynamics $(72)$, it is possible to show that the equilibrium point $x_{1}^{\circ}$ of the DAE system (1)-(64)-(70) is unstable. However the proof of this claim is omitted.

Consider now the positive definite and radially unbounded function

$$
\widetilde{W}(\eta)=\ln (1+W(\eta)),
$$

where

$$
W(\eta)=\sigma\left(E^{*}+\frac{1}{2} \eta_{1}^{2}\right),
$$

is such that (25) holds for any $c \geq 0$ and some $\sigma>0$. Note first that

$$
\left\|\frac{\partial \widetilde{W}}{\partial \eta}\right\|=\left\|\frac{\frac{\partial W}{\partial \eta}}{1+W}\right\| \leq \bar{M},
$$

for some $\bar{M}>0$. The time derivative of (74) is such that $\dot{\widetilde{W}}=\frac{\frac{\partial W}{\partial \eta} q_{0}}{1+W} \leq \frac{\gamma W+\gamma_{0}}{1+W} \leq \gamma \frac{W}{1+W}+\gamma_{0}=\gamma\left(1-\frac{1}{e^{\widetilde{W}}}\right)+\gamma_{0} \leq$ $\gamma \widetilde{W}+\left(\gamma+\gamma_{0}\right)$. Hence the function $\widetilde{W}$ satisfies both assumptions (25) and (26) of Theorem 2.

Different simulations have been carried out to evaluate the performances of the proposed stabilization methods. Consider, for instance, the case $c=0$ and the initial conditions $\theta_{1}(0)=$ $\frac{0.9999}{4} \pi, \theta_{2}(0)=\frac{3}{4} \pi, \xi_{1}=-0.0001$ and $\xi_{2}=10$. System (1)-(64)-(70) has been integrated in MATLAB by means of the solver $O D E 45$ and default absolute and relative tolerances, using the following three stabilization methods:

1) Baumgarte's method [33] with parameters $\alpha_{1}=5$ and $\alpha_{2}=25$ (equations (9)-(10));

2) the method in [38] with parameter $\gamma=10$ (equation (18)); 


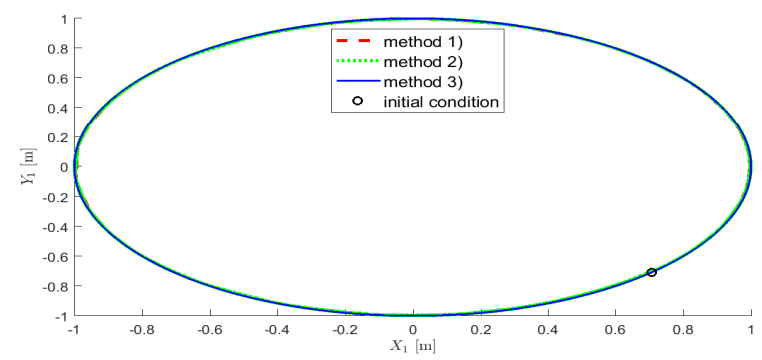

(a)

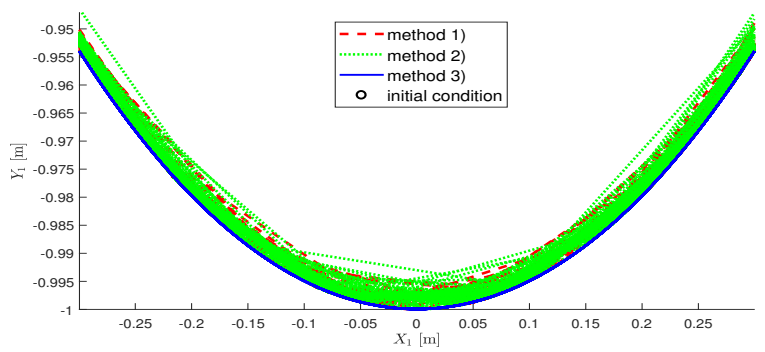

(c)

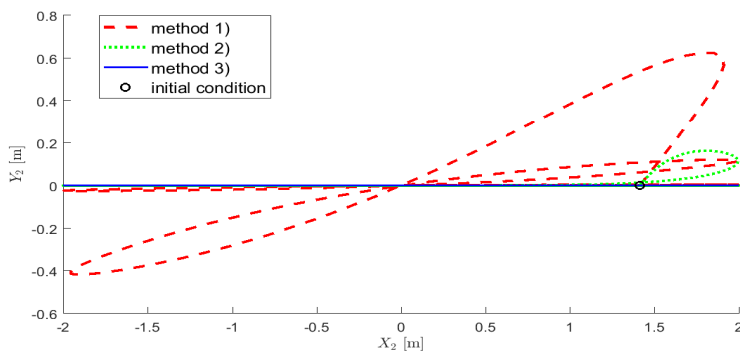

(b)

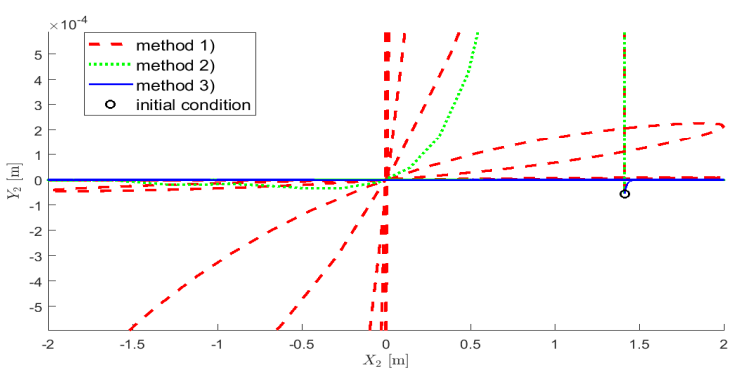

(d)

Fig. 3: Comparison of three stabilization methods (method 1 in dashed-red line, method 2 in dotted-green line and method 3 in solid-blue line) for the same initial condition (black circle): (a) trajectories of $m_{1}$ in the Cartesian plane; (b) trajectories of $m_{2}$ in the Cartesian plane; (c) zoomed in view of (a); (d) zoomed in view of (b).

3) the proposed method with parameters $\delta=1$ and $\epsilon=0.1$ (equations (21)-(22)).

Simulation results are plotted in Figures 2 and 3. In particular, in the subplots (a) and (b) of Figure 2 it is shown that, for this choice of parameters, the trajectories diverge in finite time for both methods 1) and 2) (dashed-red and dotted-green lines, respectively), while method 3) (solid-blue line) guarantees the existence of the solution for the entire time interval. The time histories of $\xi_{1}$ and $\xi_{2}$ are shown in the subplots (c) and (d), respectively, of the same figure. We observe that the trajectory obtained with method 3) (solid-blue line) has a faster transient response than the trajectories obtained with methods 1) and 2) (dashed-red and dotted-green lines, respectively). Note that a faster transient response could be achieved using methods 1) and 2) with a different choice of the parameters. However, for any fixed choice of these parameters there exists a sufficiently large inconsistent initial condition such that the corresponding trajectory diverges in finite time. The trajectories in the Cartesian plane of the masses $m_{1}$ and $m_{2}$ are shown in the subplots (a) and (b) of Figure 3, along with their zoomed in views in (c) and (d) respectively, which reveal a greater numerical accuracy for method 3). System (45)(64)-(70) has been discretized with the schemes (45) and (52) proposed in Section IV in combination with the forward Euler scheme for the zero dynamics (equation (48)). Simulations have been carried out with the same set of parameters and initial conditions as the previous one. In this setting, it is observed that condition (49) of Theorem 5 is satisfied for all $T \leq \bar{T}=0.0001$. A comparison of the trajectories obtained with the two proposed discretization schemes, for different values of the sampling time $T$, is shown in Figure 4 . Time histories of $\xi_{1}$ and $\xi_{2}$ obtained with scheme (45) are shown in the subplots (a) and (b), while the same variables obtained with scheme (52) are in the subplots (c) and (d) of the same figure. As expected, constraints stabilization is ensured for $T \leq 0.0001$ when scheme (45) is used. For $0.0001<T \leq 0.002$ stabilization of $\xi_{2}$ is still achieved with good accuracy (see (b)) while $\xi_{1}$ diverges for $T=0.002$ (see (a)). On the other hand, when scheme (52) is used, constraint stabilization in ensured for any $T>0$, see (c) and (d). Consider now the case $c=3$, i.e. the equilibrium $x_{2}^{\circ}$ is locally asymptotically stable. Let

$$
\begin{aligned}
U= & \left\{(\xi, \eta): \xi_{1} \in[-\pi, \pi], \xi_{2} \in[-20,20],\right. \\
& \left.\eta_{1} \in[-\pi, \pi], \eta_{2} \in[-4,4]\right\},
\end{aligned}
$$

and consider again the function (75). In this setting, condition (25) of Theorem 2 is satisfied, for instance, for the parameters $\gamma_{0}=0$, and for any $\gamma \geq-0.001$. Moreover, for any selection of the parameter $-\gamma>\beta>0$ there exists some $\sigma>0$ in (75) such that $\bar{M}$ in equation (32) is arbitrarily small. It is observed that condition (49) of Theorem 5 is satisfied for any $T<\bar{T}$ where $\bar{T}=0.0033$. In addition, condition (56) of Theorem 7 is satisfied for any $T<\overline{\bar{T}}$ where $\overline{\bar{T}}=0.0045$ while for any $T>0$ there exists some $\sigma>0$ such that condition (57) holds for $\bar{M}$ arbitrarily small. Simulations have been carried out by integrating system (45)-(64)-(70) with the discretization scheme (52) in combination with the forward Euler scheme for the zero dynamics (equation (48)), for the following sets of initial conditions:

1) $\theta_{1}(0)=\frac{1}{4} \pi, \theta_{2}(0)=\frac{3 \pi}{4}, \xi_{1}(0)=0, \xi_{2}(0)=0$, which are consistent with the constraint equations;

2) $\theta_{1}(0)=\frac{1}{4} \pi, \theta_{2}(0)=\frac{3}{4} \pi, \xi_{1}(0)=\frac{1}{2} \pi, \xi_{2}(0)=-4$, which are inconsistent with the constraint equations but 


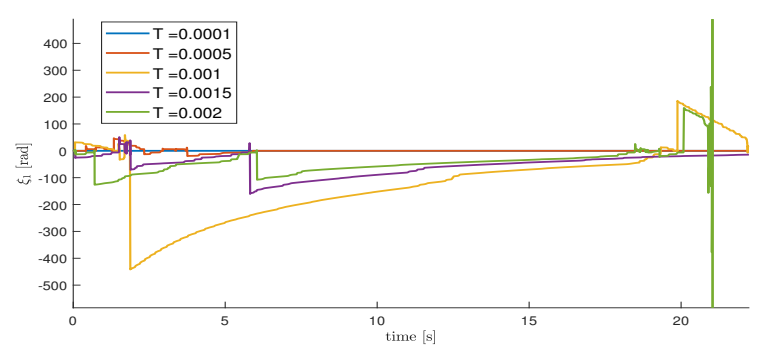

(a)

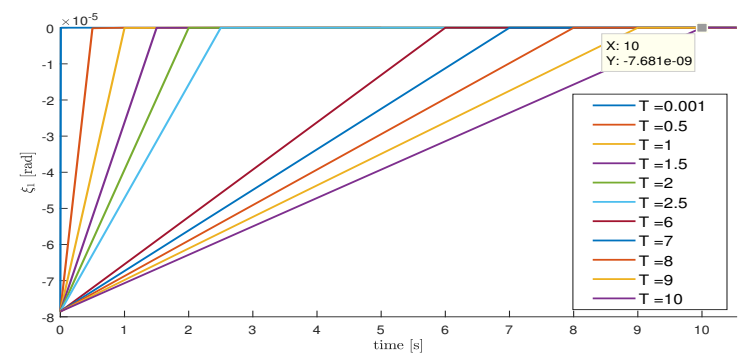

(c)

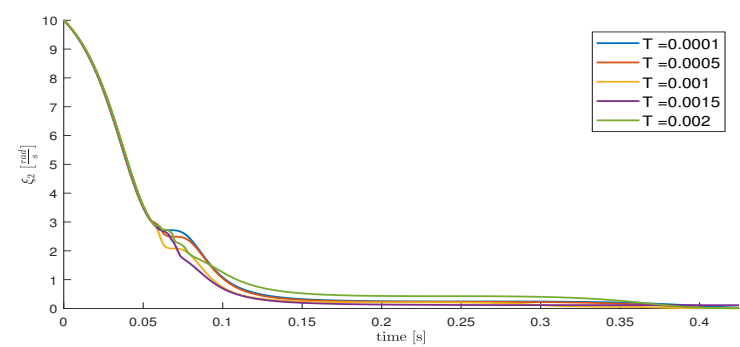

(b)

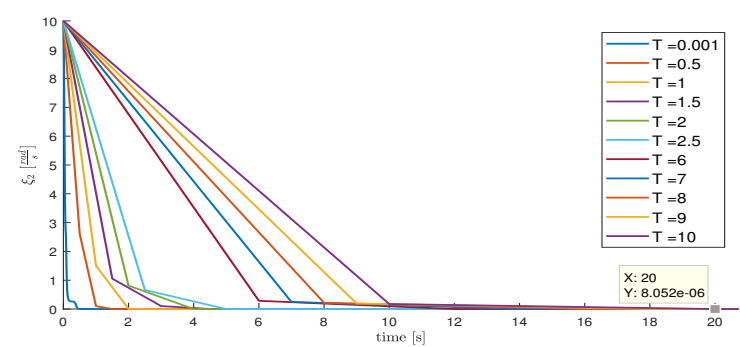

(d)

Fig. 4: Comparison of discretization schemes for different sampling times: (a) time histories of $\xi_{1}$ with the scheme (45); (b) time histories of $\xi_{2}$ with the scheme (45); (c) time histories of $\xi_{1}$ with the scheme (52); (d) time histories of $\xi_{2}$ with the scheme (52).

belong to the set $U$ in (76);

3) $\theta_{1}(0)=\frac{\pi}{4}, \theta_{2}(0)=\frac{3}{4} \pi, \xi_{1}(0)=\frac{\pi}{2}, \xi_{2}(0)=-9$, which are inconsistent and do not belong to the set $U$ in (76).

Simulation results are shown in Figure 5. Note first that for the initial condition in 1) the trajectories of $\theta_{1}$ and $\theta_{2}$ converge to the equilibrium $x_{2}^{\circ}$ as expected, see the solid line in the subplots (a) and (b). When an error in the initial condition is introduced the behaviour of the trajectories might be unpredictable. In particular, the convergence to the equilibrium $x_{2}^{\circ}$ for the initial condition in 2) is ensured by Theorem 7 (see the dashed line in the subplots (a) and (b)), while for the initial condition in 3) the trajectory converges to another equilibrium point (see the dotted line in the subplots (a) and (b)). The trajectories of the masses $m_{1}$ and $m_{2}$ in the Cartesian plane are shown in the subplots (c) and (d) of the same figure for all the considered initial conditions. Finally, time histories of $\xi_{1}$ and $\xi_{2}$ are shown in the subplots (e) and (f).

\section{CONCLUSIONS}

In this paper we have studied the problem of integration of a general class of nonlinear DAE systems with high-index. We have shown that popular stabilization methods based on a linear feedback mechanism, such as Baumgarte's method, have some limitations resulting in the occurrence of the finite escape time phenomenon when initial conditions are inconsistent. To overcome these limitations we have proposed a method based on a nonlinear stabilization of the solution manifold which guarantees the existence of the solutions for all times. In addition, the behaviour of the trajectories outside the solution manifold has been studied and some results on their stability properties have been presented. Discretization schemes have been discussed and conditions on the sampling time to preserve the stabilizing properties of the method have also been derived. The results of the paper have been summarized into a step-bystep algorithm for the simulation of nonlinear DAE systems.

\section{REFERENCES}

[1] W. Blajer, "Index of differential-algebraic equations governing the dynamics of constrained mechanical systems," Applied Mathematical Modelling, vol. 16, no. 10, pp. 70-77, 1992.

[2] A. Kumar and P. Daoutidis, Control of Nonlinear Differential Algebraic Equation Systems: An Overview. Dordrecht: Springer Netherlands, 1998, pp. 311-344.

[3] R. Riaza, Differential-Algebraic Systems: Analytical Aspects and Circuit Applications. River Edge, NJ, USA: World Scientific Publishing Co. Pte Ltd, 2008.

[4] D. Luenberger, "Dynamic equations in descriptor form," IEEE Transac tions on Automatic Control, vol. 22, no. 3, pp. 312-321, Jun 1977.

[5] K. Takaba, N. Morihira, and T. Katayama, "A generalized Lyapunov theorem for descriptor system," Systems \& Control Letters, vol. 24, no. 1, pp. 49-51, Jan 1995.

[6] J. Y. Ishihara and M. H. Terra, "On the Lyapunov theorem for singular systems," IEEE Transactions on Automatic Control, vol. 47, no. 11, pp. 1926-1930, Nov 2002.

[7] J. C. Arceo, M. Sanchez, V. Estrada-Manzo, and M. Bernal, "Convex stability analysis of nonlinear singular systems via linear matrix inequalities," IEEE Transactions on Automatic Control, pp. 1-1, 2018.

[8] N. H. McClamroch and D. Wang, "Feedback stabilization and tracking of constrained robots," IEEE Transactions on Automatic Control, vol. 33, no. 5, pp. 419-426, May 1988.

[9] N. H. McClamroch, "Feedback stabilization of control systems described by a class of nonlinear differential-algebraic equations," Systems \& Control Letters, vol. 15, no. 1, pp. 53 - 60, 1990.

[10] T. Berger, "The zero dynamics form for nonlinear differential-algebraic systems," IEEE Transactions on Automatic Control, vol. 62, no. 8, pp. 4131-4137, Aug 2017.

[11] — , "Controlled invariance for nonlinear differential-algebraic systems," Automatica, vol. 64, pp. $226-233,2016$.

[12] V. Mehrmann, "Index concepts for differential-algebraic equations," in Encyclopedia of Applied and Computational Mathematics, Engquist, B., Ed. Springer Berlin Heidelberg, 2015, pp. 676-681.

[13] C. W. Gear, Numerical Initial Value Problems in Ordinary Differential Equations. Upper Saddle River, NJ, USA: Prentice Hall PTR, 1971. 


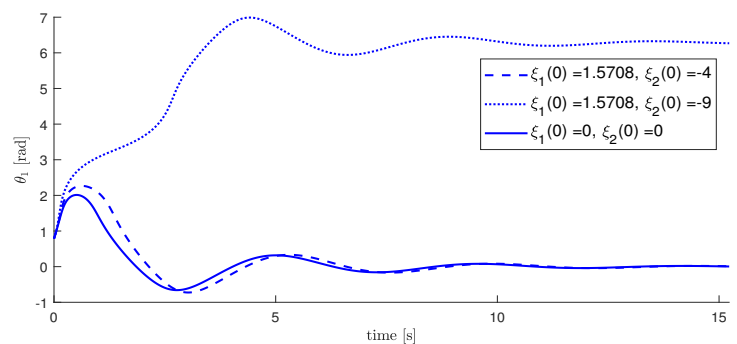

(a)

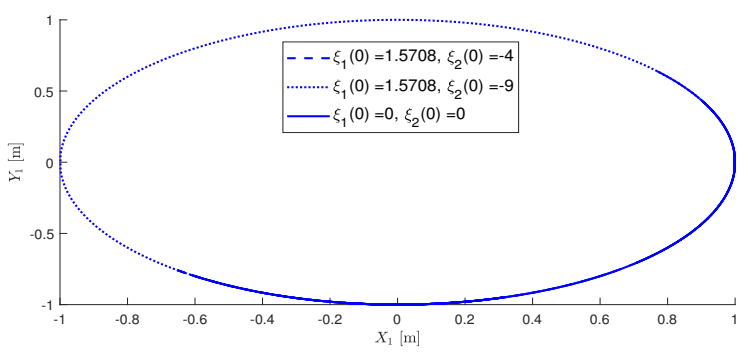

(c)

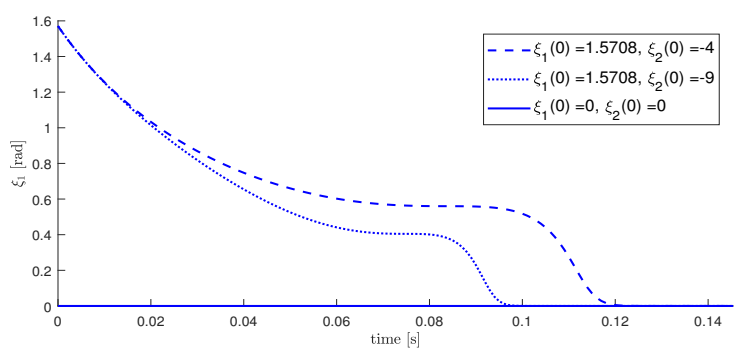

(e)

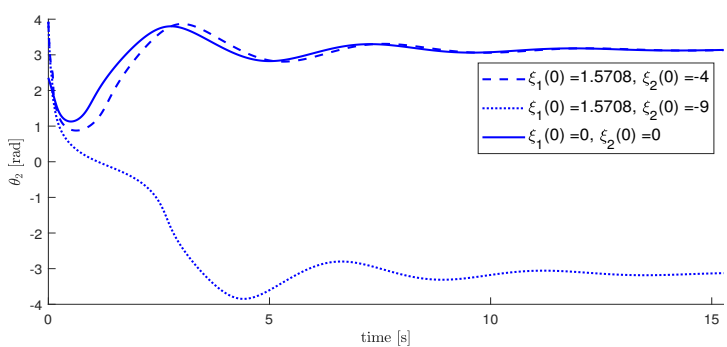

(b)

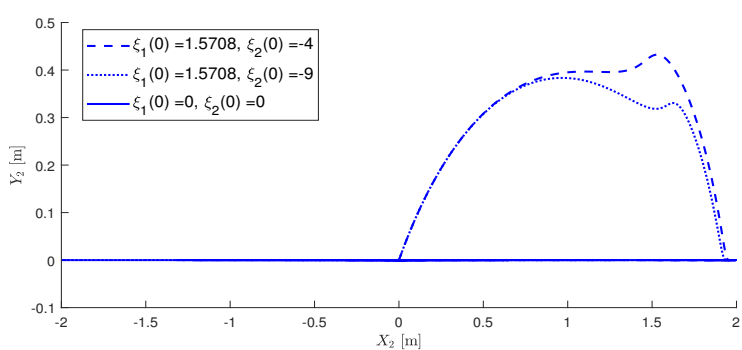

(d)

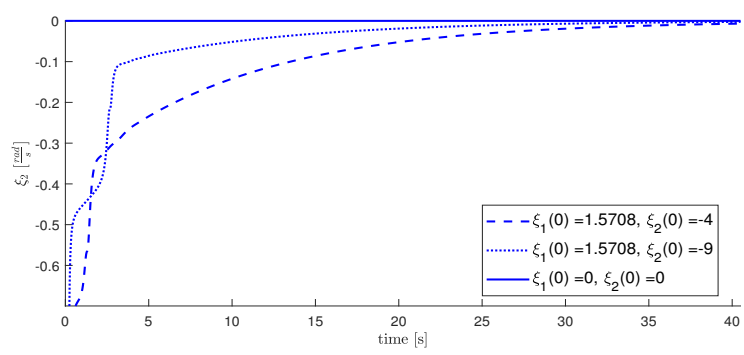

(f)

Fig. 5: Simulation results in presence of friction for different sets of initial conditions (consistent initial conditions in solid line, inconsistent initial conditions which satisfy Theorem 7 in dashed line, inconsistent initial conditions which do not satisfy Theorem 7 in dotted line): (a) time history of $\theta_{1}$; (b) time history of $\theta_{2}$; (c) trajectory of $m_{1}$ in a Cartesian plane; (d) trajectory of $m_{2}$ in a Cartesian plane; (e) time history of $\xi_{1}$; (f) time history of $\xi_{2}$.

[14] C. W. Gear and L. R. Petzold, "ODE methods for the solution of differential/algebraic systems," SIAM Journal on Numerical Analysis, vol. 21 , no. 4 , pp. 716-728, 1984.

[15] I. T. Cameron, "Solution of differential-algebraic systems using diagonally implicit Runge-Kutta methods," IMA Journal of Numerical Analysis, vol. 3, no. 3, pp. 273-289, 1983.

[16] A. Laulusa and O. A. Bauchau, "Review of classical approaches for constraint enforcement in multibody systems," Journal of Computational and Nonlinear Dynamics, vol. 3, no. 1, pp. 011004 1-8, January 2008.

[17] O. A. Bauchau. and A. Laulusa, "Review of contemporary approaches for constraint enforcement in multibody systems," Journal of Computational and Nonlinear Dynamics, vol. 3, no. 1, pp. 011005 1-8, January 2008

[18] G. A. Maggi, Principii della Teoria Matematica del Movimento Dei Corpi: Corso di Meccanica Razionale (in Italian). Milano, Italy: Ulrico Hoepli, 1896.

[19] J. I. Neimark and N. A. Fufaev, Dynamics of Nonholonomic Systems. Providence, RI: American Mathematical Society, 1972.

[20] A. Kurdila, M. P. Kamat, and J. G. Papastavridis, "Role of Maggi's equations in computational methods for constrained multibody systems,' Journal of Guidance Control and Dynamics, vol. 13, pp. 113-120, 1990.

[21] P. Lötstedt and L. Petzold, "Numerical solution of nonlinear differentia equations with algebraic constraints I: convergence results for backward differentiation formulas," Mathematics of Computation, vol. 46, no. 174, pp. 491-516, 1986.

[22] H. Hemami and F. C. Weimer, "Modeling of nonholonomic dynamic systems with applications," Journal of Applied Mechanics, vol. 48, pp 177-182, 1981.
[23] P. Ltstedt, "Mechanical systems of rigid bodies subject to unilateral constraints," SIAM Journal on Applied Mathematics, vol. 42, no. 2, pp. 281-296, 1982

[24] C. Gear, B. Leimkuhler, and G. Gupta, "Automatic integration of EulerLagrange equations with constraints," Journal of Computational and Applied Mathematics, vol. 12, pp. 77-90, 1985.

[25] F. E. Udwadia and R. E. Kalaba, "A new perspective on constrained motion," Proceedings of the Royal Society of London, vol. 439, no. 1906, pp. 407-410, 1992

[26] R. E. Kalaba and F. E. Udwadia, "On constrained motion," Applied Mathematics and Computation, vol. 51, pp. 85-86, 1992.

[27] D. C. Tarraf and H. H. Asada, "On the nature and stability of differentialalgebraic systems," in Proceedings of the 2002 American Control Conference, vol. 5, May 2002, pp. 3546-3551.

[28] S. E. Mattsson and G. Söderlind, "Index reduction in differential-algebraic equations using dummy derivatives," SIAM J. Sci. Comput., vol. 14, no. 3, pp. 677-692, May 1993.

[29] C. Pantelides, "The consistent initialization of differential-algebraic systems," SIAM Journal on Scientific and Statistical Computing, vol. 9 no. 2, pp. 213-231, 1988

[30] R. M. H. S. Yoon and D. Greenwood, "Geometric elimination of constraint violations in numerical simulation of Lagrangian equations,' Journal of Mechanical Design, vol. 116, pp. 1058-1064, 1994.

[31] W. Blajer, "Elimination of constraint violation and accuracy aspects in numerical simulation of multibody systems," Multibody System Dynamics, vol. 7, no. 3, pp. 265-284, Apr 2002.

[32] M. Arnold, B. Burgermeister, and A. Eichberger, "Linearly implicit time 
integration methods in real-time applications: DAEs and stiff ODEs," Multibody System Dynamics, vol. 17, pp. 99-117, Jan 2007.

[33] J. Baumgarte, "Stabilization of constraints and integrals of motion in dynamical systems," Computer Methods in Applied Mechanics and Engineering, vol. 1, no. 1, pp. 1-16, 1972.

[34] I. Hafner and C. Pöll, "Comparison of regularisation methods referring to a multi-pendulum case study," IFAC-PapersOnLine, vol. 48, no. 1, pp. 314-315, 2015, 8th Vienna International Conference on Mathematical Modelling.

[35] C. Pöll and I. Hafner, "Index reduction and regularisation methods for multibody systems," IFAC-PapersOnLine, vol. 48, no. 1, pp. 306-311, 2015, 8th Vienna International Conference on Mathematical Modelling.

[36] C. O. Chang and P. E. Nikravesh, "An adaptive constraint violation stabilization method for dynamic analysis of mechanical systems," Journal of Mechanisms, Transmissions, and Automation in Design, vol. 107, pp. 488-492, 1985

[37] E. J. Haug and R. C. Deyo, Eds., On Baumgarte Stabilization for Differential Algebraic Equations. Berlin, Heidelberg: Springer Berlin Heidelberg, 1991, pp. 193-207.

[38] U. Ascher, H. Chin, and S. Reich, "Stabilization of DAEs and invariant manifolds," Numerische Mathematik, vol. 67, no. 2, pp. 131-149, Mar 1994.

[39] A. Kurdila, N. Fitz-Coy, D. McDaniel, and G. Webb, "Lie algebraic control for the stabilization of nonlinear multibody system dynamics simulation," Nonlinear Dynamics, vol. 20, no. 1, pp. 55-84, Sep 1999.

[40] D. E. Chang, F. Jiménez, and M. Perlmutter, "Feedback integrators," Journal of Nonlinear Science, vol. 26, no. 6, pp. 1693-1721, Dec 2016.

[41] A. Isidori, Nonlinear Control Systems, 3rd ed., M. Thoma, E. D. Sontag, B. W. Dickinson, A. Fettweis, J. L. Massey, and J. W. Modestino, Eds. Springer-Verlag New York, Inc., 1995.

[42] P. Di Franco, G. Scarciotti, and A. Astolfi, "On the stability of constrained mechanical systems," in 2017 IEEE 56th Annual Conference on Decision and Control (CDC), Dec 2017, pp. 3170-3174.

[43] _ "A globally stable convergent algorithm for the integration of constrained mechanical systems," in 2018 American Control Conference.

[44] _ "Discretization schemes for constraint stabilization in nonlinear differential-algebraic systems," in 2018 European Control Conference.

[45] C. W. Gear, "Differential-algebraic equation index transformations," SIAM Journal on Scientific and Statistical Computing, vol. 9, no. 1, pp. 39-47, 1988.

[46] K. Brenan, S. Campbell, and L. Petzold, Numerical solution of initialvalue problems in differential-algebraic equations, ser. Classics in applied mathematics. Society for Industrial and Applied Mathematics, 1995.

[47] M. Arnold, "DAE aspects of multibody system dynamics," in Surveys in Differential-Algebraic Equations IV, A. Ilchmann and T. Reis, Eds., Jan 2017.

[48] C. Yang, J. Sun, Q. Zhang, and X. Ma, "Lyapunov stability and strong passivity analysis for nonlinear descriptor systems," IEEE Transactions on Circuits and Systems I: Regular Papers, vol. 60, no. 4, pp. 1003-1012, Apr 2013.

[49] G. D. Wood and D. C. Kennedy, "Simulating mechanical systems in Simulink with SimMechanics," 2003.

[50] J. Nestruev, Smooth Manifolds and Observables. New York: Springer, Jan 2003, vol. 220

[51] S. Monaco and D. Normand-Cyrot, "Minimum-phase nonlinear discretetime systems and feedback stabilization," in 26th IEEE Conference on Decision and Control, vol. 26, Dec 1987, pp. 979-986.

[52] S. Monaco and D. Normand-Cyrot, "Zero dynamics of sampled nonlinear systems," Systems \& Control Letters, vol. 11, no. 3, pp. 229 - 234, 1988

[53] J. LaSalle, "Some extensions of Liapunov's second method," IRE Transactions on Circuit Theory, vol. 7, no. 4, pp. 520-527, Dec 1960.

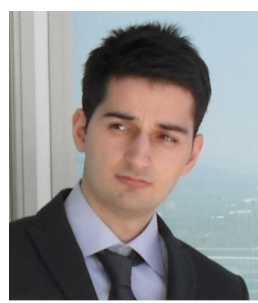

Pierluigi Di Franco was born in Melfi (Potenza) Italy, in 1988. He received the bachelor's and master's degrees in Automation Engineering from the University of Rome Tor Vergata, Italy, in 2012 and 2015, respectively. He has been a visiting student at the Electrical and Electronic Engineering Department, Imperial College of London, UK (2013-2014, 2015) where he is currently a Ph.D. student in the Control and Power Group. His research interests are focused on numerical methods, stability analysis and control of nonlinear differential-algebraic systems and applications.

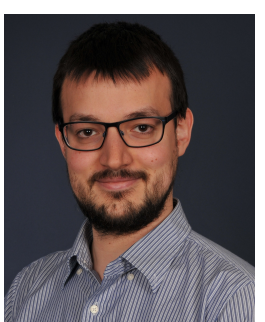

Giordano Scarciotti was born in Frascati (Rome), Italy, in 1988. He received his B.Sc. and M.Sc. degree in Automation Engineering from the University of Rome Tor Vergata, Italy, in 2010 and 2012, respectively. In 2012 he joined the Control and Power Group, Imperial College London, London, UK, where he obtained a Ph.D. degree in 2016 with a thesis on approximation, analysis and control of large-scale systems. He is currently a Lecturer (Assistant Professor) in the Control and Power Group. His current research interests are focused on analysis and control of uncertain systems (modeled by stochastic equations and set-valued mappings), the problem of model reduction and problems of optimal control. He was a visiting scholar at New York University in 2015 and at University of California Santa Barbara in 2016. He is the recipient of an Imperial College Junior Research Fellowship (2016), of the IET Control \& Automation PhD Award (2016), the Eryl Cadwaladr Davies Prize (2017) and an ItalyMadeMe award (2017).

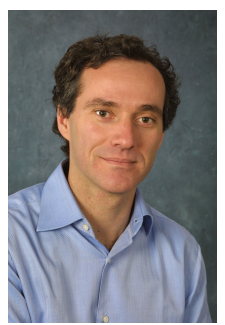

Alessandro Astolfi was born in Rome, Italy, in 1967. He graduated in electrical engineering from the University of Rome in 1991. In 1992 he joined ETH-Zurich where he obtained a M.Sc. in Information Theory in 1995 and the Ph.D. degree with Medal of Honor in 1995 with a thesis on discontinuous stabilization of nonholonomic systems. In 1996 he was awarded a Ph.D. from the University of Rome La Sapienza for his work on nonlinear robust control. Since 1996 he has been with the Electrical and Electronic Engineering Department of Imperial College London, London (UK), where he is currently Professor of Nonlinear Control Theory and Head of the Control and Power Group. From 1998 to 2003 he was also an Associate Professor at the Dept. of Electronics and Information of the Politecnico of Milano. Since 2005 he has also been a Professor at Dipartimento di Ingegneria Civile e Ingegneria Informatica, University of Rome Tor Vergata. He has been a visiting lecturer in Nonlinear Control in several universities, including ETH-Zurich (1995-1996); Terza University of Rome (1996); Rice University, Houston (1999); Kepler University, Linz (2000); SUPELEC, Paris (2001), Northeastern University (2013).

His research interests are focused on mathematical control theory and control applications, with special emphasis for the problems of discontinuous stabilization, robust and adaptive control, observer design and model reduction. He is the author of more than 150 journal papers, of 30 book chapters and of over 240 papers in refereed conference proceedings. $\mathrm{He}$ is the author (with $\mathrm{D}$. Karagiannis and R. Ortega) of the monograph "Nonlinear and Adaptive Control with Applications" (SpringerVerlag).

$\mathrm{He}$ is the recipient of the IEEE CSS A. Ruberti Young Researcher Prize (2007), the IEEE RAS Googol Best New Application Paper Award (2009), the IEEE CSS George S. Axelby Outstanding Paper Award (2012), the Automatica Best Paper Award (2017). He is a "Distinguished Member" of the IEEE CSS, IEEE Fellow and IFAC Fellow. He served as Associate Editor for Automatica, Systems and Control Letters, the IEEE Trans. on Automatic Control, the International Journal of Control, the European Journal of Control and the Journal of the Franklin Institute; as Area Editor for the Int. J. of Adaptive Control and Signal Processing; as Senior Editor for the IEEE Trans. on Automatic Control; and as Editor-inChief for the European Journal of Control. He is currently Editor-in-Chief of the IEEE Trans. on Automatic Control. He served as Chair of the IEEE CSS Conference Editorial Board (2010-2017) and in the IPC of several international conferences. He was/is a Member of the IEEE Fellow Committee (2016/2019). 